\title{
Asymptotic behaviour of global solutions to a model of cell invasion
}

\author{
Gabriela Lițcanu* and Cristian Morales-Rodrigo** \\ *Institute of Mathematics "O. Mayer", Romanian Academy, \\ 700505 Iaşi, Romania. e-mail: litcanu@uaic.ro \\ **Dpto. de Ecuaciones Diferenciales y Análisis Numérico, Universidad de Sevilla, \\ Apdo. de Correos 1160, 41080 Sevilla, Spain. e-mail: cristianm@us.es
}

\begin{abstract}
In this paper we analyze a mathematical model focusing on key events of the cells invasion process. Global well-possedness and asymptotic behaviour of nonnegative solutions to the corresponding coupled system of three nonlinear partial differential equations are studied.
\end{abstract}

Mathematics Subject Classification (2000): 35B30, 35B40, 35B45, 35K57, 35K65, 92C17 haviour

Key words or phrases: mathematical model, global existence, a priori estimates, asymptotic be-

\section{Introduction}

In this paper we focus on a mathematical model describing the process of cells invasion in the surrounding extracellular matrix. Because of the key role played by the invasive processes in biological phenomena like, for example, wound healing, morphogenesis or tumour invasion, there are a large number of studies concerning them.

In [10] the authors developed a mathematical model in order to describe the migration of tumour cells through a collagen gel. More precisely, the model is based on the hypothesis that the cells invasion is the final result of the triad of adhesion, proteolysis and motility such that in contact with the extracellular matrix, the invasive tumour cells produce proteolytic enzymes which degrade it favoring the migration. Our objective is to study a version of a model which underlies the models proposed in [3] and [10] (see also the references therein) which involves three key variables: $u(x, t)$ the density of invasive cells, $v(x, t)$ the density of extracellular matrix and $m(x, t)$ the concentration of degradative enzymes such as proteases, each of them considered at $x \in \Omega$ and time $t>0$. Through this paper $\Omega \subset \mathbb{R}^{N}, N \geqslant 1$ is a bounded domain with a regular boundary. The model is the following:

$$
\begin{array}{ll}
\frac{\partial u}{\partial t}=\underbrace{d_{1} \Delta u}_{\text {diffusion }}-\underbrace{\alpha_{1} \nabla \cdot(u \chi(v) \nabla v)}_{\text {haptotaxis }}+\underbrace{\alpha_{2} u\left(1-\alpha_{3} u-\alpha_{4} v\right)}_{\text {cells proliferation }} & x \in \Omega, \quad t \in \mathbb{R}_{+} \\
\frac{\partial v}{\partial t}=-\underbrace{\lambda m v}_{\text {degradation }} & x \in \Omega, \quad t \in \mathbb{R}_{+} \\
\frac{\partial m}{\partial t}=\underbrace{d_{2} \Delta m}_{\text {diffusion }}-\underbrace{\beta_{1} m}_{\text {decay }}+\underbrace{\beta_{2} u g(v)}_{\text {production }} & x \in \Omega, \quad t \in \mathbb{R}_{+}
\end{array}
$$

where the coefficients $d_{1}, d_{2}, \lambda, \alpha_{1}, \alpha_{3}, \alpha_{4}, \beta_{1}, \beta_{2}$ are positive constants and $\alpha_{2}$ is non-negative. It is assumed that the change in time of the cells density is due to the diffusion, to the haptotaxis with 
respect to spatial gradients in the collagen gel and to the process involving proliferation and degradation if $\alpha_{2} \neq 0$. The new cells are created at the rate $\alpha_{2}>0$ and their degradation is caused by the cells death or neutralization due to the presence of the collagen gel. The function $\chi$ describes the sensitivity of the cells to spatial gradients of collagen and is assumed to be non-negative.

In the equation (1.2) it is assumed that the proteases degrade the collagen gel at the rate $\lambda$.

The proteases concentration is affected by the diffusion, natural decay and production, this last term being proportional to the product of the cell density and a non-negative function $g$ depending on the collagen gel concentration.

In what follows we consider the system (1.1)-(1.3) together with the boundary conditions

$$
d_{1} \frac{\partial u}{\partial \eta}-\alpha_{1} u \chi(v) \frac{\partial v}{\partial \eta}=\frac{\partial m}{\partial \eta}=0, \quad x \in \partial \Omega, \quad t \in \mathbb{R}_{+}
$$

where $\eta$ denotes the unit outward normal vector of $\partial \Omega$. We have supposed that there is no flux of cells or proteases across the boundary of the domain. The same hypothesis is assumed for the extracellular matrix.

We consider also the initial conditions

$$
(u, v, m)(x, 0)=\left(u_{0}, v_{0}, m_{0}\right)(x), \quad x \in \Omega .
$$

Taking into account the biological interpretation for the solution of the system (1.1)-(1.3), we shall require that the functions $u_{0}(x), v_{0}(x)$, and $m_{0}(x)$ are non-negative.

For $g(v)=v$ and for a constant chemotactic coefficient $\chi(v)=\chi$, the mathematical model (1.1)-(1.5) was previously studied numerically in [10] assuming that the cells invasion was radially symmetrical. In the same hypotheses for $g$ and $\chi$, the system was considered in [11] where the existence of global solutions was investigated in the 3-dimensional case. In this paper we prove the global existence of the solutions when $g$ and $\chi$ are arbitrary functions satisfying some hypotheses that will be given later. Moreover, we also study the asymptotic behaviour of the solutions.

After nondimensionalizing the system (1.1)-(1.3) and redenoting the functions $\chi$ and $g$ if necessary, it becomes

$$
\begin{array}{ll}
\frac{\partial u}{\partial t}=\Delta u-\nabla \cdot(u \chi(v) \nabla v)+\mu u(1-u-v) & x \in \Omega, \quad t \in \mathbb{R}_{+} \\
\frac{\partial v}{\partial t}=-m v & x \in \Omega, \quad t \in \mathbb{R}_{+} \\
\frac{\partial m}{\partial t}=d \Delta m-\gamma m+u g(v) & x \in \Omega, \quad t \in \mathbb{R}_{+} \\
\frac{\partial u}{\partial \eta}-u \chi(v) \frac{\partial v}{\partial \eta}=\frac{\partial m}{\partial \eta}=0 & x \in \partial \Omega, \quad t \in \mathbb{R}_{+} \\
(u, v, m)(x, 0)=\left(u_{0}, v_{0}, m_{0}\right)(x) & x \in \Omega,
\end{array}
$$

where

$$
\mu=\left\{\begin{array}{ll}
1 & , \text { if } \alpha_{2} \neq 0 \\
0 & \text { if } \alpha_{2}=0
\end{array}, \quad d=\frac{d_{2}}{d_{1}}, \quad \gamma=\left\{\begin{array}{cl}
\beta_{1}\left(\alpha_{2}\right)^{-1} & \text {, if } \alpha_{2} \neq 0 \\
1 & \text {, if } \alpha_{2}=0
\end{array} .\right.\right.
$$

We notice that the new functions $\chi$ and $g$ are obtained from the initial ones by a rescaling that does not change their initial properties.

Finally, we mention here that we can obtain an equivalent system to (1.6)-(1.10) by making the change of variables

$$
w:=u z, \quad z:=e^{-\int_{0}^{v} \chi(s) d s} .
$$


In this way the system (1.6)-(1.10) transforms into

$$
\begin{array}{lll}
\frac{\partial w}{\partial t}=\Delta w+\chi(v) \nabla v \cdot \nabla w+\mu w\left(1-w z^{-1}-v\right)+\chi(v) w v m & x \in \Omega, & t \in \mathbb{R}_{+} \\
\frac{\partial v}{\partial t}=-m v & x \in \Omega, \quad t \in \mathbb{R}_{+} \\
\frac{\partial m}{\partial t}=d \Delta m-\gamma m+w z^{-1} g(v) & x \in \Omega, \quad t \in \mathbb{R}_{+} \\
\frac{\partial w}{\partial \eta}=\frac{\partial m}{\partial \eta}=0 & x \in \partial \Omega, \quad t \in \mathbb{R}_{+} \\
(w, v, m)(x, 0)=\left(u_{0} e^{-\int_{0}^{v_{0}} \chi(s) d s}, v_{0}, m_{0}\right)(x)=\left(w_{0}, v_{0}, m_{0}\right)(x) & x \in \Omega .
\end{array}
$$

In this paper we are concerning to prove the existence of a unique global solution of (1.6)-(1.10) and also to investigate asymptotic behaviour of the solution in a more general case when $d$ and $\gamma$ are positive constants and $\mu$ is a non-negative constant.

We mention that in the case when $g(v)=v$ and $\chi(v)=\chi$ is a constant, in [11 the authors proved the global existence of solutions of (1.12)-(1.16) in the 3-dimensional case based on a priori estimates. In order to derive $L^{p}$ estimates, they used a similar approach as in [12.

We point out that in what follows we establish that the a-priori $L^{p}$ estimates, $p \geqslant 1$, for the variable $w$ (and also for $u$ ) are uniform in time. This fact will be important when we establish the asymptotic behaviour of the solutions.

This paper is organized as follows. In Section 2 we give the notations and terminology used through the paper. In Section 3 we prove the local existence and the non-negativity of solutions for non-negative initial data using a fixed point method. In Section 4 we show that the solution constructed in the previous section can be prolonged in time until infinity when $N=3$. Section 5 is devoted to the stationary problem associated to (1.12)-(1.16). In the last Section we show the convergence to the steady-states and we obtain an explicit rate of convergence in some cases.

\section{Preliminaries and notations}

In this section we collect some tools and notations that will be used in the paper.

Let $\Omega \subset \mathbb{R}^{N}, N \geqslant 1$ be a bounded domain with smooth boundary. We are using in this paper the standard notation of function spaces. By $L^{p}(\Omega)$ and $W^{k, p}(\Omega)$ with $1 \leqslant p \leqslant \infty, k \geqslant 1$ we denote the Lebesgue spaces and respectively, Sobolev spaces of functions on $\Omega$. If $X$ is a Banach space with the norm $\|\cdot\|_{X}$, for $T>0$ we denote by $L^{p}(0, T ; X)$ the Banach space of all Bochner measurable functions $u:(0, T) \rightarrow X$ such that $\|u\|_{X} \in L^{p}(0, T)$.

Given a positive number $\nu$, we denote by $C^{\nu}(\bar{\Omega})$ the Hölder space of $[\nu]$ times continuously differentiable functions on $\bar{\Omega}$. We denote by $C^{\nu, \nu / 2}(\bar{\Omega} \times(0, T))$ the Hölder space of exponents $\nu$ and $\nu / 2$ by respect to $x$, respectively $t$ of continuous and bounded functions defined on $\bar{\Omega} \times(0, T)$. If $J$ is an interval of real numbers, the notation $C^{k}(J ; X), k \geqslant 1, k \in \mathbb{N}$ stands for the space of $k$ times continuously differentiable functions from $J$ to the Banach space $X$.

Throughout this paper we denote by $C, C_{i}(i=1,2, \ldots)$ positive constants which may vary from line to line. These positive constants will be independent of time, but we shall indicate explicitly on which other parameters they are dependent, if it will be the case.

Let $p \in(1, \infty)$ and $a, b$ be two positive constants. We denote

$$
A:=-a \Delta+b
$$

the positive self-adjoint operator with the domain defined by

$$
\mathcal{D}(A):=\left\{u \in W^{2, p}(\Omega) ; \quad \frac{\partial u}{\partial n}=0 \quad \text { on } \quad \partial \Omega\right\} .
$$


For $0 \leqslant \theta \leqslant 1$ we denote the fractional powers of the operator $A$ by $A^{\theta}: X_{p}^{\theta} \rightarrow L^{p}(\Omega)$ where the space $X_{p}^{\theta}$ is endowed with the graph norm

$$
\|u\|_{X_{p}^{\theta}}=\left\|A^{\theta} u\right\|_{L^{p}(\Omega)} .
$$

Let us mention that $X_{p}^{1}=\mathcal{D}(A), X_{p}^{0}=L^{p}(\Omega)$ and $X_{p}^{\theta_{1}}$ is continuously embedded into $X_{p}^{\theta_{2}}$ if $\theta_{1} \geqslant \theta_{2}$, moreover this embedding is compact if $\theta_{1}>\theta_{2}$. We recall some results that we shall use throughout the paper:

(i) we have the embedding properties (see [5, Theorem 1.6.1])

$$
\begin{array}{ll}
X_{p}^{\theta} \hookrightarrow W^{k, q}(\Omega), & k-\frac{N}{q}<2 \theta-\frac{N}{p}, \quad q \geqslant p \\
X_{p}^{\theta} \hookrightarrow C^{\nu}(\bar{\Omega}), & 0 \leqslant \nu<2 \theta-\frac{N}{p} ;
\end{array}
$$

(ii) for all $u \in L^{p}(\Omega), p \in(1, \infty)$ and $t>0$ there exists a positive constant $C(\theta)$ such that (see [5] Theorem 1.4.3])

$$
\left\|A^{\theta} e^{-t A} u\right\|_{L^{p}(\Omega)} \leqslant C(\theta) t^{-\theta} e^{-\delta t}\|u\|_{L^{p}(\Omega)}, \quad \theta \geqslant 0
$$

for some $\delta \in(0,1)$;

(iii) for all $u \in L^{p}(\Omega), 1 \leqslant q<p<\infty$ and $t>0$ we have

$$
\left\|A^{\beta} e^{-t A} u\right\|_{L^{p}(\Omega)} \leqslant C(\beta) t^{-\beta-\frac{N}{2}\left(\frac{1}{q}-\frac{1}{p}\right)} e^{-\delta t}\|u\|_{L^{q}(\Omega)}, \quad \beta \geqslant 0
$$

for $\delta \in(0,1)$.

Throughout this paper we assume that $\chi$ and $g$ are non-negative functions which satisfy the following conditions

$\left(H_{1}\right) \quad \chi \in C^{1}\left(\mathbb{R}_{+}\right), \chi \geqslant 0, \chi$ and $\chi^{\prime}$ are globally Lipschitz continuous with Lipschitz constants $L_{\chi}$ and $L_{\chi^{\prime}}$ respectively;

$\left(H_{2}\right) \quad g \in C^{1}\left(\mathbb{R}_{+}\right), g \geqslant 0, g$ and $g^{\prime}$ are globally Lipschitz continuous with Lipschitz constants $L_{g}$ and $L_{g^{\prime}}$ respectively.

\section{Local existence and uniqueness}

In this section we establish the existence of local in time non-negative solutions using a standard fixed point argument.

Let $p, q \in(1, \infty)$. We define $A_{1}=-\Delta+I$ and $A_{2}=-d \Delta+\gamma I$ the positive definite self-adjoint operators with the domains $\mathcal{D}\left(A_{1}\right)=X_{q}^{1}$ and respectively $\mathcal{D}\left(A_{2}\right)=X_{p}^{1}$ given by (2.1). Given $\tau>0,1<p<\infty$ and $\theta \in(0,1)$ we denote

$$
Y_{q}=C\left([0, \tau] ; W^{1, q}(\Omega)\right), \quad Z=C\left([0, \tau] ; X_{p}^{\theta}\right) .
$$

We consider the closed set

$$
B_{\rho}^{\tau, q}:=\left\{(w, v, m) \in Y_{q} \times Y_{\infty} \times Z ;\|(w, v, m)\|_{Y_{q} \times Y_{\infty} \times Z} \leqslant \rho\right\}
$$

where $\|(w, v, m)\|_{Y_{q} \times Y_{\infty} \times Z}:=\|w\|_{Y_{q}}+\|v\|_{Y_{\infty}}+\|m\|_{Z}$ and $\rho$ is a positive constant to be fixed later. 
For $t \in[0, \tau]$ and $(w, v, m) \in B_{\rho}^{\tau, q}$ fixed we define the mapping $F:=\left(F_{1}, F_{2}, F_{3}\right)$ by

$$
\begin{aligned}
& F_{1}(w, v, m)(t):=e^{-t A_{1}} w_{0}+\int_{0}^{t} e^{-(t-s) A_{1}} G_{1}(w, v, m)(s) d s, \\
& F_{2}(v, m)(t):=v_{0}-\int_{0}^{t} G_{2}(v, m)(s) d s, \\
& F_{3}(w, v)(t):=e^{-t A_{2}} m_{0}+\int_{0}^{t} e^{-(t-s) A_{2}} G_{3}(w, v)(s) d s,
\end{aligned}
$$

where we denoted

$$
\begin{aligned}
& G_{1}(w, v, m):=\chi(v) \nabla v \cdot \nabla w+(\mu+1) w-\mu w\left(w z^{-1}+v\right)+\chi(v) w v m \\
& G_{2}(v, m):=m v \\
& G_{3}(w, v):=w z^{-1} g(v) .
\end{aligned}
$$

Lemma 3.1 Let $\Omega \subset \mathbb{R}^{N}, N \geqslant 1$ be a domain with $C^{2}$ boundary and $p>N$. Given an initial value $\left(w_{0}, v_{0}, m_{0}\right) \in W^{1, q}(\Omega) \times W^{1, \infty}(\Omega) \times X_{p}^{\theta}$, where $\theta \in\left(\frac{N+p}{2 p}, 1\right]$ and $q \geqslant \frac{N p}{N+p}$ if $N \geqslant 2$, there exists $\tau_{0}>0$ (depending only on $\left\|u_{0}\right\|_{W^{1, q}(\Omega)},\left\|v_{0}\right\|_{W^{1, \infty}(\Omega)}$ and $\left.\left\|m_{0}\right\|_{X_{p}^{\theta}}\right)$ such that, for all $\tau \in\left(0, \tau_{0}\right]$ the application $F$ is a contraction from $B_{\rho}^{\tau, q}$ into itself.

Proof. We shall prove first that the closed set $B_{\rho}^{\tau, q}$ is invariant by $F$ for all $\tau \in\left(0, \tau_{0}\right]$ where $\tau_{0}$ will be chosen later. Taking $(w, v, m) \in B_{\rho}^{\tau, q}$, using the embeddings (2.2), (2.3), the estimate (2.4) and the hypothesis $(H 1)$ we estimate

$$
\begin{aligned}
& \left\|F_{1}(w, v, m)(t)\right\|_{W^{1, q}(\Omega)} \leqslant\left\|e^{-t A_{1}} w_{0}\right\|_{W^{1, q}(\Omega)}+C(\theta) \int_{0}^{t}\left\|A_{1}^{\theta} e^{-(t-s) A_{1}} G_{1}(w, v, m)\right\|_{L^{q}(\Omega)} d s \leqslant \\
& \leqslant C_{1}\left\|w_{0}\right\|_{W^{1, q}(\Omega)}+C(\theta) \int_{0}^{t}(t-s)^{-\theta}\left\|G_{1}(w, v, m)\right\|_{L^{q}(\Omega)} d s \leqslant \\
& \leqslant C_{1}\left\|w_{0}\right\|_{W^{1, q}(\Omega)}+C(\theta) \rho\left[\rho(\rho+1)\left(L_{\chi} \rho+\chi(0)\right)+\mu \rho\left(1+C_{2}(\rho)\right)+(\mu+1)\right] \tau^{1-\theta}, \quad \forall t \in[0, \tau]
\end{aligned}
$$

where $C_{2}(\rho)=e^{\rho\left[2^{-1} L_{\chi} \rho+\chi(0)\right]}$. We also have

$$
\left\|F_{2}(v, m)(t)\right\|_{W^{1, \infty}(\Omega)} \leqslant\left\|v_{0}\right\|_{W^{1, \infty}(\Omega)}+2 \rho^{2} \tau, \quad \forall t \in[0, \tau] .
$$

Taking into account the embeddings (2.2), (2.3), the estimate (2.4) and the hypothesis (H2), we obtain

$$
\begin{aligned}
& \left\|F_{3}(w, v)(t)\right\|_{X_{p}^{\theta}} \leqslant\left\|e^{-t A_{2}} m_{0}\right\|_{X_{p}^{\theta}}+C(\theta) \int_{0}^{t}\left\|A_{2}^{\theta} e^{-(t-s) A_{2}} w z^{-1} g(v)\right\|_{L^{p}(\Omega)} d s \leqslant \\
& \leqslant C_{3}\left\|m_{0}\right\|_{X_{p}^{\theta}}+C(\theta) \int_{0}^{t}(t-s)^{-\theta}\left\|w z^{-1} g(v)\right\|_{L^{p}(\Omega)} d s \leqslant \\
& \leqslant C_{3}\left\|m_{0}\right\|_{X_{p}^{\theta}}+C(\theta) C_{2}(\rho) \rho\left(L_{g} \rho+g(0)\right) \tau^{1-\theta}, \quad \forall t \in[0, \tau] .
\end{aligned}
$$

Denoting $C_{4}=\max \left\{1, C_{1}, C_{3}\right\}$, the estimates (3.1), (3.2) and (3.3) imply that there exists the constant

$$
C_{5}(\rho)=C(\theta) \rho\left[\rho(\rho+1)\left(L_{\chi} \rho+\chi(0)\right)+C_{2}(\rho)\left(L_{g} \rho+g(0)\right)+\mu \rho\left(1+C_{2}(\rho)\right)+(\mu+1)\right]
$$


such that

$$
\begin{aligned}
& \left\|F_{1}(w, v, m)\right\|_{Y_{q}}+\left\|F_{2}(v, m)\right\|_{Y_{\infty}}+\left\|F_{3}(w, v)\right\|_{Z} \leqslant \\
& \leqslant C_{4}\left(\left\|w_{0}\right\|_{W^{1, q}(\Omega)}+\left\|v_{0}\right\|_{W^{1, \infty}(\Omega)}+\left\|m_{0}\right\|_{X_{p}^{\theta}}\right)+C_{5}(\rho) \tau^{1-\theta}+2 \rho^{2} \tau
\end{aligned}
$$

provided that $(w, v, m) \in B_{\rho}^{\tau, q}$. Now we fix $\rho>2 C_{4}\left(\left\|w_{0}\right\|_{W^{1, q}(\Omega)}+\left\|v_{0}\right\|_{W^{1, \infty}(\Omega)}+\left\|m_{0}\right\|_{X_{p}^{\theta}}\right)>0$ sufficiently large and we choose $\tau_{1}>0$ small enough such that $F\left(B_{\rho}^{\tau, q}\right) \subset B_{\rho}^{\tau, q}$ for all $\tau \in\left(0, \tau_{1}\right]$.

In what follows we prove that $F$ is a contraction. Let $(w, v, m),(\bar{w}, \bar{v}, \bar{m}) \in B_{\rho}^{\tau, q}$, where $\tau \in\left(0, \tau_{1}\right]$ and $\rho$ was fixed previously. Taking into account (2.2), (2.3), (2.4) and the hypothesis $(H 1)$ we estimate

$$
\begin{aligned}
& \left\|F_{1}(w, v, m)(t)-F_{1}(\bar{w}, \bar{v}, \bar{m})(t)\right\|_{W^{1, q}(\Omega)} \leqslant \\
& \leqslant C(\theta) \int_{0}^{t}\left\|A_{1}^{\theta} e^{-(t-s) A_{1}}(\chi(v) \nabla v \cdot \nabla w-\chi(\bar{v}) \nabla \bar{w} \cdot \nabla \bar{v})\right\|_{L^{q}(\Omega)} d s+ \\
& +(\mu+1) C(\theta) \int_{0}^{t}\left\|A_{1}^{\theta} e^{-(t-s) A_{1}}(w-\bar{w})\right\|_{L^{q}(\Omega)} d s+\mu C(\theta) \int_{0}^{t}\left\|A_{1}^{\theta} e^{-(t-s) A_{1}}\left[\left(w^{2} z^{-1}-\bar{w}^{2} \bar{z}^{-1}\right)\right]\right\|_{L^{q}(\Omega)} d s+ \\
& +\mu C(\theta) \int_{0}^{t}\left\|A_{1}^{\theta} e^{-(t-s) A_{1}}(w v-\overline{w v})\right\|_{L^{q}(\Omega)} d s+\mu C(\theta) \int_{0}^{t}\left\|A_{1}^{\theta} e^{-(t-s) A_{1}}(\chi(v) w v m-\chi(\bar{v}) \overline{w v m})\right\|_{L^{q}(\Omega)} d s \leqslant \\
& \leqslant C_{6}(\rho) \tau^{1-\theta}\left[\|w-\bar{w}\|_{Y_{q}}+\|v-\bar{v}\|_{Y_{\infty}}+\|m-\bar{m}\|_{Z}\right], \quad \forall t \in[0, \tau]
\end{aligned}
$$

where

$$
C_{6}(\rho)=C(\theta)\left\{\rho\left(L_{\chi} \rho+\chi(0)\right)\left[1+\mu \rho\left(1+2 C_{2}(\rho)\right)\right]+2 \mu \rho C_{2}(\rho)+(\mu+1)\right\} .
$$

Also we obtain

$$
\left\|F_{2}(w, v, m)(t)-F_{2}(\bar{w}, \bar{v}, \bar{m})(t)\right\|_{W^{1, \infty}(\Omega)} \leqslant 2 \rho \tau\left(\|v-\bar{v}\|_{Y_{\infty}}+\|m-\bar{m}\|_{Z}\right), \quad \forall t \in[0, \tau] .
$$

Using (2.2), (2.3), (2.4) and the hypothesis (H2) we get

$$
\begin{aligned}
& \left\|F_{3}(w, v)(t)-F_{3}(\bar{w}, \bar{v})(t)\right\|_{X_{p}^{\theta}} \leqslant C(\theta) \int_{0}^{t}\left\|A_{2}^{\theta} e^{-(t-s) A_{2}}\left[w z^{-1} g(v)-\overline{w z}^{-1} g(\bar{v})\right]\right\|_{L^{p}(\Omega)} d s \leqslant \\
& \leqslant C_{7}(\rho) \tau^{1-\theta}\left[\|w-\bar{w}\|_{Y_{q}}+\|v-\bar{v}\|_{Y_{\infty}}\right], \quad \forall t \in[0, \tau]
\end{aligned}
$$

where

$$
C_{7}(\rho)=C(\theta) C_{2}(\rho)\left(L_{g} \rho+g(0)\right)(\rho+1)
$$

Thus, taking $\tau_{0}$ sufficiently small such that $\tau_{0}<\tau_{1}$, we obtain from (3.4), (3.5) and (3.6) that the mapping $F$ is a contraction from $B_{\rho}^{\tau, q}$ into itself for all $\tau \in\left(0, \tau_{0}\right]$.

We shall prove now the existence of a unique non-negative maximal solution to (1.12)-(1.16).

Theorem 3.2 Let $\Omega \subset \mathbb{R}^{N}, N \geqslant 1$ be a domain with $C^{2}$ boundary and $p>N$. Given $\left(w_{0}, v_{0}, m_{0}\right) \in$ $W^{1, q}(\Omega) \times W^{1, \infty}(\Omega) \times X_{p}^{\theta}, \theta \in\left(\frac{N+p}{2 p}, 1\right), q \geqslant \frac{N p}{N+p}$ if $N \geqslant 2$ and $r=\max \{2 q, p\}$, there exists $T>0$ (depending only on $\left\|w_{0}\right\|_{W^{1, q}(\Omega)},\left\|v_{0}\right\|_{W^{1, \infty}(\Omega)}$ and $\left\|m_{0}\right\|_{X_{p}^{\theta}}$ ) such that the problem (1.12)-(1.16) has a unique solution $(w, v, m)$ defined on an interval $[0, T) \subset \mathbb{R}$ and

$$
\begin{aligned}
w & \in C\left([0, T) ; W^{1, q}(\Omega)\right) \cap C\left((0, T) ; W^{2, r}(\Omega)\right) \cap C^{1}\left((0, T) ; W^{1, q}(\Omega)\right) \\
v & \in C\left([0, T) ; W^{1, \infty}(\Omega)\right) \cap C^{1}\left((0, T) ; W^{1, \infty}(\Omega)\right) \\
m & \in C\left([0, T) ; X_{p}^{\theta}\right) \cap C\left((0, T) ; W^{2, p}(\Omega)\right) \cap C^{1}\left((0, T) ; X_{p}^{\theta}\right) .
\end{aligned}
$$

Moreover, the solution depends continuously on the initial data. 
Proof. Lemma 3.1 shows that the map $F: B_{\rho}^{\tau, q} \rightarrow B_{\rho}^{\tau, q}, \tau \in\left(0, \tau_{0}\right]$, has a unique fixed point $(w, v, m)$ which is the weak solution to the system (1.12)-(1.16). Taking into account the fact that the map $F$ is a contraction and the estimates established in Lemma 3.1 we obtain the continuous dependence of this solution on the initial data.

In what follows we show the existence of a unique maximal solution to (1.12)-(1.16) having the regularity properties stated in the theorem. First we can prove that for every fixed $t \in\left(0, \tau_{0}\right]$ the maps $G_{1}(t)$ : $W^{1, q}(\Omega) \times W^{1, \infty}(\Omega) \times X_{p}^{\theta} \rightarrow L^{q}(\Omega), G_{2}(t): W^{1, \infty}(\Omega) \times X_{p}^{\theta} \rightarrow W^{1, \infty}(\Omega)$ and $G_{3}(t): W^{1, q}(\Omega) \times W^{1, \infty}(\Omega) \rightarrow$ $L^{p}(\Omega)$ are Lipschitzian using similar arguments to those used in the proof of Lemma 3.1. Therefore applying [5, Theorem 3.5.2] we obtain

$$
w \in C^{1}\left(\left(0, \tau_{0}\right] ; W^{1, q}(\Omega)\right), \quad m \in C^{1}\left(\left(0, \tau_{0}\right] ; X_{p}^{\theta}\right) .
$$

Let us observe that for every $t \in\left(0, \tau_{0}\right], w(t) \in W^{1, q}(\Omega)$ is the solution to the problem

$$
\begin{array}{ll}
-\Delta w(t)-a(t) \cdot \nabla w(t)=f(t)-\frac{\partial w}{\partial t}(t) & x \in \Omega \\
\frac{\partial w}{\partial \eta}=0 & x \in \partial \Omega
\end{array}
$$

where we have denoted

$$
a(t):=\chi(v(t)) \nabla v(t) \in\left(L^{\infty}(\Omega)\right)^{N}, \quad f(t):=\left(\mu w\left(1-w z^{-1}-v\right)+\chi(v) w v m\right)(t) \in L^{q}(\Omega) .
$$

The elliptic regularity implies that $w(t) \in W^{2, q}(\Omega)$ for every $t \in\left(0, \tau_{0}\right]$. As $q>N / 2$, we deduce from the Sobolev embeddings that $w(t) \in W^{2,2 q}(\Omega)$. Finally, by recurrence, we obtain $w(t) \in W^{2, r}(\Omega)$, $r=\max \{2 q, p\}$. In a similar manner we can prove

$$
m \in C\left(\left(0, \tau_{0}\right] ; W^{2, p}(\Omega)\right), \quad 2 \leqslant p<\infty .
$$

In order to show the uniqueness of the solutions in the spaces indicated above, let us suppose that $\left(w_{1}, v_{1}, m_{1}\right),\left(w_{2}, v_{2}, m_{2}\right)$ are two different solutions to the system (1.12)-(1.16). Let $[0, T)$ the maximal interval where both solutions are defined and

$$
I=\left\{t \in[0, T) ; \quad w_{1}(x, t)=w_{2}(x, t), v_{1}(x, t)=v_{2}(x, t), m_{1}(x, t)=m_{2}(x, t), \forall x \in \Omega\right\} .
$$

From the local existence we deduce that $I$ is a nonempty set, so let $\tau_{\max }>0$ be maximal such that $\left[0, \tau_{\max }\right) \subset I$ and suppose $\tau_{\max }<T$. As $I$ is a closed set in $[0, T)$ (from the continuity of the solutions), it follows that $\left[0, \tau_{\max }\right] \subset I$. Applying now the local existence result with the initial conditions considered in $\tau_{\max }$ it follows that the system (1.12)-(1.16) has a unique solution on a small interval $\left(\tau_{\max }, \tau_{\max }+\varepsilon\right)$. This contradicts the maximality of $\tau_{\max }$, hence $\tau_{\max }=T$.

Let us remark that the choice of $\tau_{0}$ in Lemma 3.1 depends only on the initial data, respectively $\left\|w_{0}\right\|_{W^{1, q}(\Omega)}$, $\left\|v_{0}\right\|_{W^{1, p}(\Omega)}$ and $\left\|m_{0}\right\|_{X_{p}^{\theta}}$, so the solution $(u, v, m)$ can be extended up to a maximal time $\tau_{\max }=T$ that depends also only on the initial data.

Remark 3.1 Let us mention that in fact we can obtain from the Theorem 3.2 a better regularity for $w$. Indeed, $G_{1}(t) \in C^{\alpha}(\Omega)$ for some $0<\alpha<1$ and $w(t, \cdot) \in C^{2+\alpha}(\Omega)$. Thus for $t>0,(t, x) \rightarrow w\left(t, x ; w_{0}\right)$ is continuously differentiable in $t$, twice continuously differentiable in $x$, and hence $w$ is a classical solution.

In view of (1.11) we observe that Theorem 3.2 implies also the local existence of the solution to the initial system (1.6)-(1.10). Moreover, we shall show the non-negativity of local solutions to (1.6)-(1.10) corresponding to non-negative initial values $\left(w_{0}, v_{0}, m_{0}\right)$.

Theorem 3.3 Let $\Omega \subset \mathbb{R}^{N}, N \geqslant 1$ be a domain with $C^{2}$ boundary and $p>N$. Given the non-negative initial value $\left(u_{0}, v_{0}, m_{0}\right) \in W^{1, p}(\Omega) \times W^{1, \infty}(\Omega) \times X_{p}^{\theta}, \theta \in\left(\frac{N+p}{2 p}, 1\right)$, there exists $T>0$ (depending only 
on $\left\|w_{0}\right\|_{W^{1, p}(\Omega)},\left\|v_{0}\right\|_{W^{1, \infty}(\Omega)}$ and $\left.\left\|m_{0}\right\|_{X_{p}^{\theta}}\right)$ such that the problem (1.6)-(1.10) has a unique non-negative solution $(u, v, m)$ defined on an interval $[0, T) \subset \mathbb{R}$ and

$$
\begin{aligned}
u & \in C\left([0, T) ; W^{1, q}(\Omega)\right) \cap C\left((0, T) ; W^{1, \infty}(\Omega)\right) \cap C^{1}\left((0, T) ; W^{1, q}(\Omega)\right) \\
v & \in C\left([0, T) ; W^{1, \infty}(\Omega)\right) \cap C^{1}\left((0, T) ; W^{1, \infty}(\Omega)\right) \\
m & \in C\left([0, T) ; X_{p}^{\theta}\right) \cap C\left((0, T) ; W^{2, p}(\Omega)\right) \cap C^{1}\left((0, T) ; X_{p}^{\theta}\right) .
\end{aligned}
$$

Moreover, the solution depends continuously on the initial data.

Proof. From (1.11) and taking into account $\left(u_{0}, v_{0}\right) \in W^{1, p}(\Omega) \times W^{1, \infty}(\Omega)$ we obtain $w_{0} \in W^{1, p}(\Omega)$ and the hypotheses of Theorem 3.2 are satisfied. Using again (1.11) we recover the regularity of $u$.

Let us observe that from the equation (1.7) we obtain

$$
v=v_{0} e^{-\int_{0}^{t} m(x, s) d s}
$$

which implies the nonnegativity of $v$. We shall prove the nonnegativity of the solution $u$ by the truncation technique used in [13, Theorem 2.1]. According to [13, there exists a decreasing function $H \in \mathcal{C}^{3}\left(\mathbb{R} ; \overline{\mathbb{R}}_{+}\right)$ and a constant $C_{56}>0$ such that $H(u)>0$ if $u<0$ and $H(u)=0$ if $u \geqslant 0$ and having the properties

$$
\begin{array}{llll}
0 \leqslant H^{\prime \prime}(u) u^{2} \leqslant \widetilde{C} H(u), & & u \in \mathbb{R} \\
0 \leqslant H^{\prime}(u) u \leqslant \widetilde{C} H(u), & & u \in \mathbb{R} \\
0 \leqslant H(u) \leqslant \widetilde{C} u^{2}, & & u \in \mathbb{R} .
\end{array}
$$

We consider the non-negative function

$$
\varphi(t)=\int_{\Omega} H(u(x, t)) d x, \quad 0 \leqslant t \leqslant \tau .
$$

The definition of the function $H(u)$ implies that $\varphi \in \mathcal{C}\left([0, \tau] ; \overline{\mathbb{R}}_{+}\right) \cap \mathcal{C}^{1}((0, \tau] ; \mathbb{R}), \varphi(0)=0$ and $\varphi$ has the derivative

$$
\begin{aligned}
\varphi^{\prime}(t) & =\int_{\Omega} H^{\prime}(u) \frac{\partial u}{\partial t} d x=-\int_{\Omega} H^{\prime \prime}(u)|\nabla u|^{2} d x+\int_{\Omega} u \chi(v) H^{\prime \prime}(u) \nabla u \cdot \nabla v d x+\mu \int_{\Omega} H^{\prime}(u) u(1-u-v) d x \leqslant \\
& \leqslant \frac{1}{2} \widetilde{C} \int_{\Omega} H(u) \chi^{2}(v)|\nabla v|^{2} d x+\mu \widetilde{C} \int_{\Omega} H(u) d x \leqslant C_{8} \varphi(t)
\end{aligned}
$$

for all $0 \leqslant t \leqslant \tau$ where

$$
C_{8}=\widetilde{C}\left[\frac{1}{2}\left(L_{\chi}\|v\|_{L^{\infty}(\Omega)}+\chi(0)\right)^{2}\|\nabla v\|_{L^{\infty}(\Omega)}^{2}+\mu\right] .
$$

Thus the Gronwall inequality ensures $\varphi(t)=0$ for all $t \in[0, \tau]$, that is $u(t) \geqslant 0$ on $\Omega$ for $t \in[0, \tau]$. Finally, since $u g(v)$ is a non-negative function it is straightforward to prove that $m(t) \geqslant 0$ on $\Omega$ for $t \in[0, \tau]$ using the maximum principle for parabolic equations. But $\tau>0$ is arbitrary, so the desired positivity follows.

Theorem 3.4 Under the same hypotheses as in Theorem 3.3. if $v_{0} \in W^{2, r}(\Omega), r=\max \{2 q, p\}$ then the problem (1.6)-(1.10) has a unique non-negative solution $(u, v, m)$ defined on an interval $[0, T) \subset \mathbb{R}$ and

$$
\begin{aligned}
u & \in C\left([0, T) ; W^{1, q}(\Omega)\right) \cap C\left((0, T) ; W^{2, r}(\Omega)\right) \cap C^{1}\left((0, T) ; W^{1, q}(\Omega)\right) \\
v & \in C\left([0, T) ; W^{2, r}(\Omega)\right) \cap C^{1}\left((0, T) ; W^{2, r}(\Omega)\right) \\
m & \in C\left([0, T) ; X_{p}^{\theta}\right) \cap C\left((0, T) ; W^{2, p}(\Omega)\right) \cap C^{1}\left((0, T) ; X_{p}^{\theta}\right) .
\end{aligned}
$$

Moreover, the solution depends continuously on the initial data. 


\section{Global existence in time}

We denote by $(u, v, m)$ the maximal non-negative local solution to the problem (1.6)-(1.10) on $[0, T)$ and we shall prove that $T=+\infty$ in the case $N=3$. Throughout this section all the constants are independent of $T$ and when it will be the case we shall make explicit their dependence on the data of the problem.

Lemma 4.1 Let $\Omega \subset \mathbb{R}^{N}, N \geqslant 1$ be a domain with smooth boundary and $\left(u_{0}, v_{0}, m_{0}\right) \in L^{1}(\Omega) \times L^{\infty}(\Omega) \times$ $L^{1}(\Omega)$. Then the solution $(u, v, m)$ to the problem (1.6)-(1.10) satisfies the following estimates

$$
\begin{aligned}
& \|u(\cdot, t)\|_{L^{1}(\Omega)} \leqslant \max \left\{|\Omega|,\left\|u_{0}\right\|_{L^{1}(\Omega)}\right\} \\
& \|v(\cdot, t)\|_{L^{\infty}(\Omega)} \leqslant\left\|v_{0}\right\|_{L^{\infty}(\Omega)}, \\
& \|m(\cdot, t)\|_{L^{1}(\Omega)} \leqslant\left\|m_{0}\right\|_{L^{1}(\Omega)} e^{-t}+\left[L_{g}\left\|v_{0}\right\|_{L^{\infty}(\Omega)}+g(0)\right] \max \left\{|\Omega|,\left\|u_{0}\right\|_{L^{1}(\Omega)}\right\}
\end{aligned}
$$

for all $t>0$.

Proof. Integrating the equation (1.6) in space and taking into account the non-negativity of the solution and the boundary condition (1.9) we get

$$
\frac{d}{d t} \int_{\Omega} u d x \leqslant \mu \int_{\Omega} u(x, t) d x-\mu \int_{\Omega} u^{2}(x, t) d x .
$$

Applying Jensen's inequality and Gronwall's lemma we obtain (4.1).

The boundedness of $v$ results immediately from the equation (1.7). We obtain (4.3) integrating the equation (1.8) in space and taking into account the boundary condition (1.9), the estimates (4.1), (4.2) and the Gronwall lemma.

Corollary 4.2 Let $\Omega \subset \mathbb{R}^{N}, N \geqslant 1$ be a domain with smooth boundary and $u_{0} \in L^{1}(\Omega)$ (or, equivalently $\left.\left(w_{0}, v_{0}\right) \in L^{1}(\Omega) \times L^{\infty}(\Omega)\right)$. Then

$$
\|w(\cdot, t)\|_{L^{1}(\Omega)} \leqslant \max \left\{|\Omega|,\left\|u_{0}\right\|_{L^{1}(\Omega)}\right\},
$$

for all $t>0$, where $w(x, t)$ is the function given by (1.11).

In what follows we show that if the initial data $m_{0}$ is in an appropriate space, then we can find a bound for $m$ in $L^{j}(\Omega), j>1$ for $t>0$. This result is based on [6, Lemma 4.1].

Lemma 4.3 Let $\Omega \subset \mathbb{R}^{N}, N \geqslant 2$ be a domain with smooth boundary. Assume that there exist $r \in[1, N)$ and $1<p<r N /(N-r)$ such that

$$
\|u(\cdot, t)\|_{L^{r}(\Omega)} \leqslant C_{9}
$$

for all $t \in(0, T)$ and $\left(v_{0}, m_{0}\right) \in L^{\infty}(\Omega) \times W^{1, p}(\Omega)$. Then

$$
\|m(\cdot, t)\|_{W^{1, p}} \leqslant C\left(p, r,\left\|v_{0}\right\|_{L^{\infty}(\Omega)},\left\|m_{0}\right\|_{W^{1, p}(\Omega)}\right)\left(1+C_{9}\right)
$$

for all $t \in(0, T)$.

Proof. We fix $1<p<r N /(N-r)$ and we choose $\beta$ such that

$$
\frac{1}{2}<\beta<\frac{1}{2}+\frac{N}{2}\left(\frac{1}{p}-\frac{N-r}{r N}\right) .
$$

From the representation formula

$$
m(t)=e^{-t A_{2}} m_{0}+\int_{0}^{t} e^{-(t-s) A_{2}} u(s) g(v(s)) d s, \quad \forall t \in(0, T)
$$


and taking into account (2.5) we obtain

$$
\begin{aligned}
& \|m(\cdot, t)\|_{W^{1, p}} \leqslant C(\beta)\left\|m_{0}\right\|_{W^{1, p}}+\int_{0}^{t} \| A_{2}^{\beta} e^{-(t-s) A_{2}} u(s) g\left(v(s) \|_{L^{p}(\Omega)} d s \leqslant\right. \\
& \leqslant C(\beta)\left\|m_{0}\right\|_{W^{1, p}}+\frac{C(\beta, p) C_{9}}{1-\beta-\frac{N}{2}\left(\frac{1}{r}-\frac{1}{p}\right)}\left[L_{g}\left\|v_{0}\right\|_{L^{\infty}(\Omega)}+g(0)\right], \quad \forall t \in(0, T)
\end{aligned}
$$

Using Lemma 4.1 the statement follows.

Remark 4.1 If $m_{0} \in L^{1}(\Omega)$, the estimate 4.5) is still valid for all $t \in[\tau, T)$ where $\tau \in(0, \min \{1, T\})$.

Corollary 4.4 Let $\Omega \subset \mathbb{R}^{N}, N>2$ be a domain with smooth boundary and assume that the hypotheses of Lemma 4.3 are satisfied. If $r \in[1, N / 2)$ then

$$
\|m(\cdot, t)\|_{L^{j}} \leqslant C_{10}
$$

for all $t \in(0, T)$ and $\frac{N}{N-1}<j<\frac{r N}{N-2 r}$.

The next proposition asserts that if the initial value is in a suitable space and (4.7) is satisfied, then we obtain an estimate for $w$, and respectively for $u$, in $L^{q}(\Omega)$, for all $q>1$ and $t>0$. Moreover, we shall show later that if $w_{0} \in L^{\infty}(\Omega)$ then we derive a bound for $w$ in $L^{\infty}(\Omega)$ for all $t>0$.

Proposition 4.5 Let $\Omega \subset \mathbb{R}^{3}$ be a domain with smooth boundary and $\left(w_{0}, v_{0}, m_{0}\right) \in L^{q}(\Omega) \times L^{\infty}(\Omega) \times$ $W^{1, p}, 1<q<\infty, p \geqslant \frac{6}{5}$. Then there exists a constant independent on time $C_{11}=C_{11}\left(p,\left\|w_{0}\right\|_{L^{q}(\Omega)},\left\|v_{0}\right\|_{L^{\infty}(\Omega)}\right)$ such that the solution $w(x, t)$ to the system (1.12)-(1.16) satisfies

$$
\|w\|_{L^{\infty}\left(0, T ; L^{q}(\Omega)\right)} \leqslant C_{11}, \quad \forall 1<q<+\infty .
$$

Proof. Multiplying the equation (1.12) with $q w^{q-1} z^{-1}, q \in[2, \infty)$ and integrating in space we have

$$
\begin{aligned}
\frac{d}{d t} \int_{\Omega} z^{-1} w^{q} & =-\frac{4(q-1)}{q} \int_{\Omega} z^{-1}\left|\nabla w^{q / 2}\right|^{2}+\mu q \int_{\Omega} z^{-1} w^{q}\left(1-w z^{-1}-v\right)+ \\
& +(q-1) \int_{\Omega} z^{-1} \chi(v) w^{q} v m .
\end{aligned}
$$

On both sides of (4.9) we add the term $\varepsilon\left\|w^{q / 2}\right\|_{L^{2}(\Omega)}^{2}$ where $\varepsilon>0$ is a constant to be determined later. Taking into account the non-negativity of the solution and Lemma 4.1 we obtain

$$
\begin{aligned}
\frac{d}{d t} \int_{\Omega} z^{-1} w^{q}+\varepsilon\left\|w^{q / 2}\right\|_{L^{2}(\Omega)}^{2} & \leqslant-\frac{4(q-1)}{q} \int_{\Omega} z^{-1}\left|\nabla w^{q / 2}\right|^{2}+\left(q \mu C_{12}+\varepsilon\right)\left\|w^{q / 2}\right\|_{L^{2}(\Omega)}^{2}+ \\
& +(q-1) C_{13} \int_{\Omega} w^{q} m
\end{aligned}
$$

where

$$
\begin{aligned}
& C_{12}=C_{12}\left(\left\|v_{0}\right\|_{L^{\infty}(\Omega)}\right)=e^{\frac{L_{\chi}}{2}\left\|v_{0}\right\|_{L^{\infty}(\Omega)}^{2}+\chi(0)\left\|v_{0}\right\|_{L^{\infty}(\Omega)}}, \\
& C_{13}=C_{13}\left(\left\|v_{0}\right\|_{L^{\infty}(\Omega)}\right)=e^{\frac{L_{\chi}}{2}\left\|v_{0}\right\|_{L^{\infty}(\Omega)}^{2}+\chi(0)\left\|v_{0}\right\|_{L^{\infty}(\Omega)}}\left(L_{\chi}\left\|v_{0}\right\|_{L^{\infty}(\Omega)}+\chi(0)\right)\left\|v_{0}\right\|_{L^{\infty}(\Omega)} .
\end{aligned}
$$

Now we estimate the last two terms from the right-hand side of (4.10). Using Gagliardo-Nirenberg and Young's inequalities and Lemma 4.1 we obtain

$$
\begin{aligned}
\left(q \mu C_{12}+\varepsilon\right)\left\|w^{q / 2}\right\|_{L^{2}(\Omega)}^{2} & \leqslant C(\Omega)\left(q \mu C_{12}+\varepsilon\right)\left\|w^{q / 2}\right\|_{W^{1,2}(\Omega)}^{6(q-1) /(3 q-1)}\|w\|_{L^{1}(\Omega)}^{2 q /(3 q-1)} \leqslant \\
& \leqslant \frac{\varepsilon}{4}\left\|w^{q / 2}\right\|_{W^{1,2}(\Omega)}^{2}+C_{14}\|w\|_{L^{1}(\Omega)}^{4 q /(3 q-1)}
\end{aligned}
$$


where

$$
C_{14}=C_{14}\left(\varepsilon, q,\left\|v_{0}\right\|_{L^{\infty}(\Omega)},|\Omega|\right)=\left(\frac{2}{3(q-1)}\right)\left(\frac{\varepsilon}{4}\right)^{-3(q-1) / 2}\left(\frac{3(q-1)}{3 q-1} C(\Omega)\left(q \mu C_{12}+\varepsilon\right)\right)^{(3 q-1) / 2} .
$$

Taking into account Hölder, Gagliardo-Nirenberg and Young's inequalities, the boundedness (4.7) and Lemma 4.1 we estimate the last term from the right-hand side of (4.10)

$$
\begin{aligned}
(q-1) C_{13} \int_{\Omega} w^{q} m & \leqslant(q-1) C_{13}\left\|w^{q / 2}\right\|_{L^{4}(\Omega)}^{2}\|m\|_{L^{2}(\Omega)} \leqslant \\
& \leqslant(q-1) C_{13} C(\Omega)\left\|w^{q / 2}\right\|_{W^{1,2}(\Omega)}^{3(2 q-1) /(3 q-1)}\left\|w^{q / 2}\right\|_{L^{2 / q}(\Omega)}^{2 /(3 q-1)}\|m\|_{L^{2}(\Omega)} \leqslant \\
& \leqslant \frac{\varepsilon}{4}\left\|w^{q / 2}\right\|_{W^{1,2}(\Omega)}^{2}+C_{15}\|m\|_{L^{2}(\Omega)}^{4(3 q-1)}\|w\|_{L^{1}(\Omega)}^{q},
\end{aligned}
$$

where

$$
C_{15}=C_{15}\left(\varepsilon, q,\left\|v_{0}\right\|_{L^{\infty}(\Omega)},|\Omega|\right)=\left(\frac{1}{3(2 q-1)}\right)\left(\frac{\varepsilon}{4}\right)^{-3(2 q-1)}\left(\frac{3(2 q-1)}{2(3 q-1)}(q-1) C_{13} C(\Omega)\right)^{2(3 q-1)} .
$$

From (4.10), using (4.4), (4.7), (4.11) and (4.12), we get

$$
\begin{aligned}
& \frac{d}{d t} \int_{\Omega} z^{-1} w^{q}+\varepsilon\left\|w^{q / 2}\right\|_{L^{2}(\Omega)}^{2} \leqslant \\
& \leqslant-\frac{4(q-1)}{q} \int_{\Omega} z^{-1}\left|\nabla w^{q / 2}\right|^{2}+\frac{\varepsilon}{2}\left\|w^{q / 2}\right\|_{W^{1,2}(\Omega)}^{2}+C_{14}(\varepsilon)\|w\|_{L^{1}(\Omega)}^{4 q /(3 q-1)}+C_{15}(\varepsilon)\|m\|_{L^{2}(\Omega)}^{4(3 q-1)}\|w\|_{L^{1}(\Omega)}^{q} \leqslant \\
& \leqslant\left(\frac{\varepsilon}{2}-\frac{4(q-1)}{q}\right)\left\|\nabla w^{q / 2}\right\|_{L^{2}(\Omega)}^{2}+\frac{\varepsilon}{2}\left\|w^{q / 2}\right\|_{L^{2}(\Omega)}^{2}+C_{16}
\end{aligned}
$$

where

$$
C_{16}=C_{16}\left(\varepsilon, q,\left\|v_{0}\right\|_{L^{\infty}(\Omega)},|\Omega|\right)=\left(C_{14}(\varepsilon, q)+C_{15}(\varepsilon, q) C_{10}^{4(3 q-1)}\right)\left(\max \left\{1,|\Omega|,\left\|u_{0}\right\|_{L^{1}(\Omega)}\right\}\right)^{q} .
$$

Choosing $\varepsilon=2$, from (4.13) we obtain

$$
\frac{d}{d t} \int_{\Omega} z^{-1} w^{q}+\frac{1}{C_{12}} \int_{\Omega} z^{-1} w^{q} \leqslant C_{16}
$$

Applying Gronwall's lemma, the last inequality implies

$$
\|w\|_{L^{q}(\Omega)}^{q} \leqslant \int_{\Omega} z^{-1} w^{q} \leqslant C_{12} \max \left\{\left\|w_{0}\right\|_{L^{q}(\Omega)}^{q}, C_{16}\right\}
$$

and we conclude the proof.

Corollary 4.6 Let $\Omega \subset \mathbb{R}^{3}$ be a domain with smooth boundary and $\left(u_{0}, v_{0}, m_{0}\right) \in L^{q}(\Omega) \times L^{\infty}(\Omega) \times W^{1, p}$, $1<q<\infty, p \geqslant \frac{6}{5}$. Then there exists a constant $C_{17}=C_{17}\left(p,\left\|u_{0}\right\|_{L^{q}(\Omega)},\left\|v_{0}\right\|_{L^{\infty}(\Omega)},\left\|m_{0}\right\|_{W^{1, p}(\Omega)}\right)$ independent on time such that the solution $u(x, t)$ to the system (1.12)-(1.16) satisfies

$$
\|u\|_{L^{\infty}\left(0, T ; L^{q}(\Omega)\right)} \leqslant C_{17}, \quad \forall 1<q<+\infty .
$$

We prove now the uniform boundedness for $w$ using the previous Proposition. For this we use the iterative technique of Alikakos [1] and for the sake of completeness this argument will be presented briefly in the next Proposition. 
Proposition 4.7 Let $\Omega \subset \mathbb{R}^{3}$ be a domain with smooth boundary and $\left(w_{0}, v_{0}, m_{0}\right) \in L^{\infty}(\Omega) \times L^{\infty}(\Omega) \times$ $W^{1, p}, p \geqslant \frac{6}{5}$. Then there exists a constant $C_{18}=C_{18}\left(\left\|w_{0}\right\|_{L^{\infty}(\Omega)},\left\|v_{0}\right\|_{L^{\infty}(\Omega)}\right)$ independent on time such that the solution $w(x, t)$ to the system (1.12)-(1.16) satisfies

$$
\|w\|_{L^{\infty}\left(0, T ; L^{\infty}(\Omega)\right)} \leqslant C_{18} .
$$

Proof. We estimate the last term from the right-hand side of (4.10). Using Hölder, Gagliardo-Nirenberg and Young's inequalities and (4.7)

$$
\begin{aligned}
(q-1) C_{13} \int_{\Omega} w^{q} m & \leqslant(q-1) C_{13}\left\|w^{q / 2}\right\|_{L^{4}(\Omega)}^{2}\|m\|_{L^{2}(\Omega)} \leqslant \\
& \leqslant(q-1) C_{13} C(\Omega)\left\|w^{q / 2}\right\|_{W^{1,2}(\Omega)}^{9 / 5}\left\|w^{q / 2}\right\|_{L^{1}(\Omega)}^{1 / 5}\|m\|_{L^{2}(\Omega)} \leqslant \\
& \leqslant \varepsilon\left\|\nabla w^{q / 2}\right\|_{L^{2}(\Omega)}^{2}+\varepsilon\left\|w^{q / 2}\right\|_{L^{2}(\Omega)}^{2}+C_{19}(\varepsilon, q)\left\|w^{q / 2}\right\|_{L^{1}(\Omega)}^{2},
\end{aligned}
$$

where

$$
C_{19}=C_{19}(\varepsilon, q)=\frac{1}{9}\left(\frac{9}{10}\right)^{10} \varepsilon^{-9}\left[(q-1) C_{13} C(\Omega)\right]^{10}\|m\|_{L^{2}(\Omega)}^{10} .
$$

Introducing (4.16) in (4.10) we obtain

$$
\begin{aligned}
\frac{d}{d t} \int_{\Omega} z^{-1} w^{q}+\varepsilon\left\|w^{q / 2}\right\|_{L^{2}(\Omega)}^{2} & \leqslant\left[\varepsilon-\frac{4(q-1)}{p}\right] \int_{\Omega}\left|\nabla w^{q / 2}\right|^{2}+ \\
& +\left(\mu q C_{12}+2 \varepsilon\right)\left\|w^{q / 2}\right\|_{L^{2}(\Omega)}^{2}+C_{19}(\varepsilon, q)\left\|w^{q / 2}\right\|_{L^{1}(\Omega)}^{2}
\end{aligned}
$$

Using again Gagliardo-Nirenberg and Young's inequalities, we infer

$$
\begin{aligned}
\left\|w^{q / 2}\right\|_{L^{2}(\Omega)}^{2} & \leqslant C_{20}(\Omega)\left\|w^{q / 2}\right\|_{W^{1,2}(\Omega)}^{6 / 5}\left\|w^{q / 2}\right\|_{L^{1}(\Omega)}^{4 / 5} \leqslant \\
& \leqslant \varepsilon\left\|\nabla w^{q / 2}\right\|_{L^{2}(\Omega)}^{2}+\varepsilon\left\|w^{q / 2}\right\|_{L^{2}(\Omega)}^{2}+C_{21}(\varepsilon)\left\|w^{q / 2}\right\|_{L^{1}(\Omega)}^{2},
\end{aligned}
$$

or equivalently

$$
\left\|w^{q / 2}\right\|_{L^{2}(\Omega)}^{2} \leqslant \frac{1}{1-\varepsilon}\left[\varepsilon\left\|\nabla w^{q / 2}\right\|_{L^{2}(\Omega)}^{2}+C_{21}(\varepsilon)\left\|w^{q / 2}\right\|_{L^{1}(\Omega)}^{2}\right]
$$

where $\varepsilon<1$ and

$$
C_{21}=C_{21}(\varepsilon)=\frac{2}{3}\left(\frac{3}{5}\right)^{5 / 2} \varepsilon^{-\frac{3}{2}} C_{20}^{5 / 2}(\Omega)
$$

Multiplying the inequality (4.18) by $\left(\mu q C_{12}+2 \varepsilon\right)$, we obtain from (4.17)

$$
\begin{aligned}
\frac{d}{d t} \int_{\Omega} z^{-1} w^{q}+\varepsilon\left\|w^{q / 2}\right\|_{L^{2}(\Omega)}^{2} & \leqslant\left[\varepsilon-\frac{4(q-1)}{q}\right] \int_{\Omega}\left|\nabla w^{q / 2}\right|^{2}+C_{19}(\varepsilon, q)\left\|w^{q / 2}\right\|_{L^{1}(\Omega)}^{2}+ \\
& +\frac{\left(\mu q C_{12}+2 \varepsilon\right)}{1-\varepsilon}\left[\varepsilon\left\|\nabla w^{q / 2}\right\|_{L^{2}(\Omega)}^{2}+C_{21}\left\|w^{q / 2}\right\|_{L^{1}(\Omega)}^{2}\right]= \\
& =\left[\varepsilon+\frac{\left(\mu q C_{12}+2 \varepsilon\right)}{1-\varepsilon} \varepsilon-\frac{4(q-1)}{q}\right] \int\left|\nabla w^{q / 2}\right|^{2}+ \\
& +\left[\frac{\left(\mu q C_{12}+2 \epsilon\right)}{1-\varepsilon} C_{21}+C_{19}(\varepsilon, q)\right]\left\|w^{q / 2}\right\|_{L^{1}(\Omega)}^{2} .
\end{aligned}
$$


We choose

$$
\varepsilon=\frac{1}{C_{22}(q+1)}<1
$$

where $C_{22}=\max \left\{\mu C_{12}, 3\right\}$. Hence

$$
\varepsilon+\frac{\left(\mu q C_{12}+2 \varepsilon\right)}{1-\varepsilon} \varepsilon<\frac{4(q-1)}{q} .
$$

Thus we obtain from (4.19)

$$
\frac{d}{d t} \int_{\Omega} z^{-1} w^{q}+\frac{1}{C_{12} C_{22}(q+1)} \int_{\Omega} z^{-1} w^{q} \leqslant C_{23}(q)\left\|w^{q / 2}\right\|_{L^{1}(\Omega)}^{2},
$$

where

$$
\begin{aligned}
C_{23} & =C_{23}(\varepsilon, q)=\frac{2}{3}\left(\frac{3}{5}\right)^{5 / 2} C_{20}^{5 / 2}(\Omega) \frac{\left(\mu q C_{12} C_{22}(q+1)+2\right)}{C_{22}(q+1)-1}\left(C_{22}(q+1)\right)^{\frac{3}{2}}+ \\
& +\frac{1}{9}\left(\frac{9}{10}\right)^{10}\left(C_{22}(q+1)\right)^{9}\left[(q-1) C_{13} C(\Omega)\right]^{10}\|m\|_{L^{2}(\Omega)}^{10} .
\end{aligned}
$$

Applying Gronwall's lemma we deduce from the last inequality

$$
\int_{\Omega} w^{q} \leqslant \int_{\Omega} z^{-1} w^{q} \leqslant C_{12} \max \left\{\int_{\Omega} w_{0}^{q}, C_{22} C_{23}(\varepsilon, q)(q+1)\left(\sup _{t \geqslant 0} \int_{\Omega} w^{q / 2}\right)^{2}\right\} .
$$

We consider $q_{j}=2^{j}$, with $j \in \mathbb{N}$ and we use (4.21) and an iterative technique in order to estimate $\|w\|_{L^{q_{j}}(\Omega)}$. Denoting

$$
a_{j}=C_{22} C_{23}(\varepsilon, q)(q+1) \leqslant C_{24} q_{j}^{20},
$$

the inequality (4.21) becomes

$$
\int_{\Omega} w^{q_{j}} \leqslant C_{12} \max \left\{\max \left\{\left\|w_{0}\right\|_{L^{1}(\Omega)}^{q_{j}},\left\|w_{0}\right\|_{L^{\infty}(\Omega)}^{q_{j}}\right\}, a_{j}\left(\sup _{t \geqslant 0} \int_{\Omega} w^{q_{j} / 2}\right)^{2}\right\}
$$

for all $t>0$. Recursively, we obtain from (4.22)

$$
\begin{aligned}
\|w\|_{L^{q_{j}}} & \leqslant C_{12}^{1 / q_{j}} \max \left\{\left\|w_{0}\right\|_{L^{1}(\Omega)},\left\|w_{0}\right\|_{L^{\infty}(\Omega)}\right\}\left(a_{j} a_{j-1}^{q_{1}} a_{j-2}^{q_{2}} \ldots a_{1}^{q_{j-1}}\right)^{\frac{1}{q_{j}}} \leqslant \\
& \leqslant 2^{20 \sum_{k=1}^{j} \frac{j}{2^{j}}} C_{12}^{\frac{1}{2^{j}}} C_{24}^{\left(1-\frac{1}{2^{j}}\right)} \max \left\{\left\|w_{0}\right\|_{L^{1}(\Omega)},\left\|w_{0}\right\|_{L^{\infty}(\Omega)}\right\} .
\end{aligned}
$$

Taking $j \rightarrow \infty$ in the last inequality we deduce (4.15).

Corollary 4.8 Let $\Omega \subset \mathbb{R}^{3}$ be a domain with smooth boundary and $\left(u_{0}, v_{0}, m_{0}\right) \in L^{\infty}(\Omega) \times L^{\infty}(\Omega) \times$ $W^{1, p}(\Omega), p \geqslant \frac{6}{5}$. Then there exists a constant $C_{25}=C_{25}\left(\left\|u_{0}\right\|_{L^{\infty}(\Omega)},\left\|v_{0}\right\|_{L^{\infty}(\Omega)}\right)$ independent on time such that the solution $u$ to the system (1.12)-(1.16) satisfies

$$
\|u\|_{L^{\infty}\left(0, T ; L^{\infty}(\Omega)\right)} \leqslant C_{25} .
$$

Lemma 4.9 Let $\Omega \subset \mathbb{R}^{3}$ be a domain with smooth boundary and $\left(u_{0}, v_{0}, m_{0}\right) \in L^{p}(\Omega) \times L^{\infty}(\Omega) \times X_{p}^{\theta}(\Omega)$ where $p>3, \theta \in\left(\frac{1}{2}, 1\right)$. Then

$$
\|m(\cdot, t)\|_{X_{p}^{\theta}} \leqslant C_{26}
$$

for all $t \in(0, T)$. 
Proof. Taking into account the representation formula (4.6), the hypothesis $\left(H_{2}\right)$ and the estimate (4.14) we obtain

$$
\begin{aligned}
\|m(\cdot, t)\|_{X_{p}^{\theta}} & \leqslant C\left\|m_{0}\right\|_{X_{p}^{\theta}}+\int_{0}^{t}\left\|A_{2}^{\theta} e^{-(t-s) A_{2}} u(s) g(v(s))\right\|_{L^{p}} d s \leqslant \\
& \leqslant C\left\|m_{0}\right\|_{X_{p}^{\theta}}+C(\theta) \int_{0}^{t}(t-s)^{-\theta} e^{-\delta(t-s)}\|u(s) g(v(s))\|_{L^{p}} d s \leqslant \\
& \leqslant C\left\|m_{0}\right\|_{X_{p}^{\theta}}+C_{17} C(\theta)\left(L_{g}\left\|v_{0}\right\|_{L^{\infty}}+g(0)\right) \int_{0}^{t}(t-s)^{-\theta} e^{-\delta(t-s)} d s .
\end{aligned}
$$

Denoting

$$
C_{26}=\max \left\{C\left\|m_{0}\right\|_{X_{p}^{\theta}}, C_{17} C(\theta)\left(L_{g}\left\|v_{0}\right\|_{L^{\infty}}+g(0)\right) \delta^{\theta-1} \Gamma(1-\theta)\right\},
$$

where $\Gamma$ denotes the Gamma function, the last inequality implies (4.25).

Lemma 4.10 Let $\Omega \subset \mathbb{R}^{3}$ be a domain with smooth boundary and $\left(u_{0}, v_{0}, m_{0}\right) \in L^{p}(\Omega) \times W^{1, \infty}(\Omega) \times$ $X_{p}^{\theta}(\Omega)$ where $p>3, \theta \in\left(\frac{3+p}{2 p}, 1\right)$. Then

$$
\|v(t)\|_{W^{1, \infty}} \leqslant C_{27}(1+t)
$$

for all $t \in(0, T)$.

Proof. From the equation (1.13) we obtain

$$
\nabla v=e^{-\int_{0}^{t} m}\left(\nabla v_{0}-v_{0} \int_{0}^{t} \nabla m\right)
$$

which implies

$$
\|\nabla v\|_{L^{\infty}} \leqslant\left\|\nabla v_{0}\right\|_{L^{\infty}}+\left\|v_{0}\right\|_{L^{\infty}} \int_{0}^{t}\|m\|_{W^{1, \infty}} d s \leqslant\left\|v_{0}\right\|_{w^{1, \infty}} \max \left\{1, C_{26}\right\}(1+t) .
$$

Next, taking into account Lemma 4.9 and denoting

$$
C_{27}=\left\|v_{0}\right\|_{L^{\infty}(\Omega)} \max \left\{1, C_{26}\right\}
$$

we conclude the proof.

Lemma 4.11 Let $\Omega \subset \mathbb{R}^{3}$ be a domain with smooth boundary and $\left(u_{0}, v_{0}, m_{0}\right) \in W^{1, q}(\Omega) \times W^{1, \infty}(\Omega) \times$ $X_{p}^{\theta}(\Omega)$ where $p>3, \theta \in\left(\frac{3+p}{2 p}, 1\right)$ and $q \geqslant \frac{3 p}{p+3}$. For $t \in(0, T)$ we have

$$
\|u(\cdot, t)\|_{W^{1, q}(\Omega)} \leqslant C_{28}(1+t) e^{C_{29} t},
$$

for all $t \in(0, T)$.

Proof. First we establish a bound for $\|w(\cdot, t)\|_{W^{1, q}(\Omega)}$. Taking into account the representation formula

$$
w(x, t)=e^{-t A_{1}} w_{0}+\int_{0}^{t} e^{-(t-s) A_{1}} G_{1}(w, v, m)(s) d s, \quad \forall t \in(0, T)
$$


we get

$$
\begin{aligned}
& \|w(\cdot, t)\|_{W^{1, q}(\Omega)} \leqslant\left\|e^{-t A_{1}} w_{0}\right\|_{W^{1, q}(\Omega)}+\int_{0}^{t}\left\|A_{1}^{\theta} e^{-(t-s) A_{1}} G_{1}(w, v, m)\right\|_{L^{q}(\Omega)} d s \leqslant \\
& \leqslant C\left\|w_{0}\right\|_{W^{1, q}(\Omega)}+C(\theta, q) C_{27}\left[L_{\chi}\left\|v_{0}\right\|_{L^{\infty}(\Omega)}+\chi(0)\right] \int_{0}^{t}(t-s)^{-\theta} e^{-\delta(t-s)}(1+s)\|w\|_{W^{1, q}(\Omega)} d s+ \\
& +C_{11}\left[(\mu+1)+\mu C_{11} C_{12}+\mu\left\|v_{0}\right\|_{L^{\infty}(\Omega)}\right] \int_{0}^{t}(t-s)^{-\theta} e^{-\delta(t-s)} d s+ \\
& +C_{11} C_{26} L_{\chi}\left\|v_{0}\right\|_{L^{\infty}(\Omega)}\left[\left\|v_{0}\right\|_{L^{\infty}(\Omega)}+\chi(0)\right] \int_{0}^{t}(t-s)^{-\theta} e^{-\delta(t-s)} d s \leqslant \\
& \leqslant C_{30}+C_{31} \int_{0}^{t}(t-s)^{-\theta} e^{-\delta(t-s)}(1+s)\|w\|_{W^{1, q}(\Omega)} d s
\end{aligned}
$$

where

$$
\begin{aligned}
C_{30}=C\left\|w_{0}\right\|_{W^{1, q}(\Omega)}+C_{11} \delta^{\theta-1} \max \left\{C_{26}\left\|v_{0}\right\|_{L^{\infty}(\Omega)}\left[L_{\chi}\left\|v_{0}\right\|_{L^{\infty}(\Omega)}+\chi(0)\right],\right. & \\
& {\left.\left[(\mu+1)+\mu C_{11} C_{12}+\mu\left\|v_{0}\right\|_{L^{\infty}(\Omega)}\right]\right\} \Gamma(1-\theta), } \\
C_{31} & =C(\theta, q) C_{27}\left[L_{\chi}\left\|v_{0}\right\|_{L^{\infty}(\Omega)}+\chi(0)\right] .
\end{aligned}
$$

and $\Gamma$ is the Gamma function. Applying Gronwall's lemma in the last inequality we obtain

$$
\|w(\cdot, t)\|_{W^{1, q}(\Omega)} \leqslant C_{30} e^{\Gamma(1-\theta) C_{31}} e^{\Gamma(1-\theta) C_{31} t} .
$$

Finally, as $u=w z^{-1}$ we get from (4.26) and (4.30)

$$
\begin{aligned}
\|u(\cdot, t)\|_{W^{1, q}(\Omega)} & \leqslant\left\|z^{-1}\right\|_{W^{1, \infty}(\Omega)}\|w\|_{W^{1, q}(\Omega)} \leqslant \\
& \leqslant C_{12}\left[1+C_{27}\left(L_{\chi}\left\|v_{0}\right\|_{L^{\infty}(\Omega)}+\chi(0)\right)(1+t)\right]\|w\|_{W^{1, q}(\Omega)} \leqslant \\
& \leqslant 2 C_{12} C_{30} \max \left\{1, C_{27}\left(L_{\chi}\left\|v_{0}\right\|_{L^{\infty}(\Omega)}+\chi(0)\right)\right\} e^{\Gamma(1-\theta) C_{31}}(1+t) e^{\Gamma(1-\theta) C_{31} t} .
\end{aligned}
$$

Denoting

$$
\begin{aligned}
& C_{28}=2 C_{12} C_{30} \max \left\{1, C_{27}\left(L_{\chi}\left\|v_{0}\right\|_{L^{\infty}(\Omega)}+\chi(0)\right)\right\} e^{C_{31} \Gamma(1-\theta)}, \\
& C_{29}=C_{31} \Gamma(1-\theta)
\end{aligned}
$$

the last inequality implies (4.28).

\section{$5 \quad$ Steady-states}

Additionally, we suppose that the function $g$ satisfies the following property:

$$
\left(H_{3}\right) \quad g(v) \neq 0 \text { if } v \neq 0 .
$$


In this section we deal with the stationary problem associated to (1.12)-(1.16). More precisely, we are interested in the solution of the system

$$
\begin{array}{ll}
0=\Delta w+\chi(v) \nabla v \cdot \nabla w+\mu w\left(1-w z^{-1}-v\right) & x \in \Omega \\
0=m v & x \in \Omega \\
0=d \Delta m-\gamma m+w z^{-1} g(v) & x \in \Omega \\
\frac{\partial w}{\partial \eta}=\frac{\partial m}{\partial \eta}=0 & x \in \partial \Omega .
\end{array}
$$

Theorem 5.1 If $\left(w^{*}, v^{*}, m^{*}\right) \in C^{1}(\bar{\Omega}) \times W^{1, \infty}(\Omega) \times C^{1}(\bar{\Omega})$ are the non-negative solutions to (5.1)-(5.4), then they are given by

$$
\begin{aligned}
\left(w^{*}, v^{*}, m^{*}\right) & =(0, \widetilde{v}, 0), \\
\left(w^{*}, v^{*}, m^{*}\right) & =\left(k, 0, k \gamma^{-1} g(0)\right)
\end{aligned}
$$

where $k=0$ or $k=1$ if $\mu>0, k \geqslant 0$ is an arbitrary constant if $\mu=0$ and $\widetilde{v} \in W^{1, \infty}(\Omega)$ is an arbitrary non-negative function.

Proof. We distinguish between two cases: $w(x) g(v(x))>0$ at some point $x \in \Omega$, or $w g(v) \equiv 0$. First, we suppose that there exists at least a point $x \in \Omega$ such that $w(x) g(v(x)) \neq 0$. We claim that

$$
\min _{x \in \bar{\Omega}} m(x)>0 .
$$

To prove this, we assume that there exists $x_{0} \in \bar{\Omega}$ such that $m\left(x_{0}\right)=0$. We have two possibilities:

a. if $x_{0} \in \Omega$, then [4, Theorem 3.5] implies that $m$ is a constant function and we deduce that $m(x) \equiv 0$ for all $x \in \Omega$, but this is not a solution for the equation (5.3).

b. if $x_{0} \in \partial \Omega$, then using [4, Lemma 3.4] we get

$$
\frac{\partial m}{\partial \eta}\left(x_{0}\right)<0
$$

which contradicts (5.4).

Since $\min _{x \in \bar{\Omega}} m(x)>0$, the equation (5.2) implies $v(x) \equiv 0$ for all $x \in \Omega$. Therefore the function $w(x)$ is a solution of the following equation

$$
\begin{cases}-\Delta w=\mu w(1-w) & x \in \Omega \\ \frac{\partial w}{\partial \eta}=0 & x \in \partial \Omega .\end{cases}
$$

a. If $\mu=0$, we deduce that the solutions of (5.5) are $w \equiv k$ where $k$ is a non-negative constant.

b. If $\mu>0$, then using a similar argument as in [2] we obtain that $w \equiv 0$ and $w \equiv 1$ are the only non-negative solutions to (5.5).

Therefore, from (5.3)-(5.4) we obtain that $m$ is a constant and moreover

$$
m \equiv k \gamma^{-1} g(0)
$$

where $k$ is a non-negative constant if $\mu=0$ and $k=0$ or $k=1$ if $\mu>0$.

We suppose now that for all $x \in \Omega, w g(v) \equiv 0$. Then from (5.3)-(5.4) we get $m \equiv 0$.

We observe that $w g(v) \equiv 0$ implies $w v \equiv 0$, using the hypothesis $\left(H_{3}\right)$. Arguing as in the previous case we prove that either $w \equiv 0$ or $\min _{x \in \bar{\Omega}} w(x)>0$. 
a. If $w \equiv 0$ then any $\widetilde{v} \in W^{1, \infty}(\Omega), \widetilde{v} \geqslant 0$ solves (5.2).

b. If $\min _{x \in \bar{\Omega}} w(x)>0$ then $w g(v) \equiv 0$ implies $g(v) \equiv 0$ and by $\left(H_{3}\right) v \equiv 0$. Therefore, (5.1) with the boundary condition can be written in the form (5.5). Hence $w \equiv k$ where $k$ is a non-negative constant if $\mu=0$ and $k=0$ or $k=1$ if $\mu>0$.

\section{Asymptotic behaviour of global solutions}

In what follows we study the asymptotic behaviour of the global solution $(u, v, m)$ to the problem (1.6)(1.10). Under some additionally hypotheses on the initial data we shall prove the convergence of the solutions to the steady states.

Hereafter $\Omega \subset \mathbb{R}^{3}$ is a domain with smooth boundary.

Lemma 6.1 Let $\mu \geqslant 0, v_{0} \in L^{\infty}(\Omega)$ be positive and if $\mu>0$ we assume $0<v_{0}(x)<1$ for all $x \in \Omega$. If there exists a constant $a>0$ such that $u_{0}(x) \geqslant a$, then every global solution $u(x, t)$ satisfies

$$
u(x, t) \geqslant \min \{1, a\} e^{\int_{0}^{v_{0}} \chi(s) d s},
$$

for all $x \in \Omega, t>0$. Moreover, if there exists a positive constant $M$ such that

$$
g(v) \geqslant M
$$

for all $v \in \mathbb{R}_{+}$, then there exists a constant $\sigma>0$ such that

$$
m(x, t) \geqslant \sigma>0
$$

for all $x \in \Omega, t>0$.

Proof. Let $\rho$ be a positive constant to be chosen later. By multiplying the equation (1.6) with $z^{-1}(w-$ $\rho)_{-}$, where $(w-\rho)_{-}=\max \{\rho-w, 0\}$, and after that integrating over $\Omega$ we get

$$
\begin{aligned}
\frac{1}{2} \frac{d}{d t} \int_{\Omega}\left[z^{-1}(w-\rho)_{-}^{2}\right] & =-\int_{\Omega} z^{-1}\left|\nabla(w-\rho)_{-}\right|^{2}-\frac{1}{2} \int_{\Omega} z^{-1} \chi(v) m v(w-\rho)_{-}^{2}- \\
& -\int_{\Omega} z^{-1} \chi(v) m v w(w-\rho)_{-}-\mu \int_{\Omega} z^{-1} w\left(1-w z^{-1}-v\right)(w-\rho)_{-} .
\end{aligned}
$$

Let us notice that in the case $\mu=0$ the right-hand side of (6.4) is non-positive. If $\mu>0$, choosing the constant $0<\rho \leqslant\left(1-v_{0}\right) e^{-\int_{0}^{v_{0}} \chi(s) d s}$ implies that the last term in (6.4) is also non-positive. Integrating (6.4) in time and taking into account the above considerations we have

$$
\int_{\Omega} z^{-1}(w-\rho)_{-}^{2} \leqslant \int_{\Omega} e^{\int_{0}^{v_{0}} \chi(s) d s}\left(w_{0}-\rho\right)_{-}^{2} .
$$

Using the same procedure as in 8 finally we obtain

$$
u(x, t) \geqslant \min \{1, a\} e^{\int_{0}^{v_{0}} \chi(s) d s},
$$


for all $x \in \Omega, t>0$. Next, we conclude the proof by applying the maximum principle for the parabolic problem

$$
\begin{array}{ll}
m_{t}-d \Delta m+\gamma m=u g(v) & x \in \Omega, \quad t \in \mathbb{R}_{+} \\
\frac{\partial m}{\partial \eta}=0 & x \in \partial \Omega, \quad t \in \mathbb{R}_{+} \\
m(x, 0)=m_{0}(x) & x \in \Omega
\end{array}
$$

using the hypothesis $g(v) \geqslant M>0$ and the estimate (6.5).

Lemma 6.2 Let $\left(u_{0}, v_{0}, m_{0}\right) \in L^{1}(\Omega) \times\left(W^{1,2}(\Omega) \cap L^{\infty}(\Omega)\right) \times W^{1, p}(\Omega), p \geqslant \frac{6}{5}$. Assume that $v_{0}>0$ and the assumption (6.2) is satisfied. Then we have

$$
\int_{\Omega}\left|\nabla\left(v^{q / 2}(t)\right)\right|^{2} \leqslant C_{33} e^{-k t}
$$

for all $t>0, q \geqslant 1$ and $0<k<q \sigma$.

Proof. Using the equation (1.7) we deduce

$$
\frac{d}{d t} \int_{\Omega}\left|\nabla v^{q / 2}\right|^{2}=-q \int_{\Omega} m\left|\nabla v^{q / 2}\right|^{2}-\frac{q}{2} \int_{\Omega} \nabla\left(v^{q}\right) \cdot \nabla m,
$$

for $q \geqslant 1$. On the other hand, multiplying (1.8) with $v^{q}, q \geqslant 1$ and integrating in space, we have

$$
-\frac{d}{2} \int_{\Omega} \nabla m \cdot \nabla\left(v^{q}\right)=\frac{1}{2} \frac{d}{d t} \int_{\Omega} m v^{q}+\frac{q}{2} \int_{\Omega} m^{2} v^{q}+\frac{\gamma}{2} \int_{\Omega} m v^{q}-\frac{1}{2} \int_{\Omega} u g(v) v^{q} .
$$

Inserting (6.8) in (6.7) we get

$$
\frac{d}{d t} \int_{\Omega}\left|\nabla v^{q / 2}\right|^{2}=-q \int_{\Omega} m\left|\nabla v^{q / 2}\right|^{2}+\frac{q}{2 d} \frac{d}{d t} \int_{\Omega} m v^{q}+\frac{q^{2}}{2 d} \int_{\Omega} m^{2} v^{q}+\frac{\gamma q}{2 d} \int_{\Omega} m v^{q}-\frac{q}{2 d} \int_{\Omega} u g(v) v^{q} .
$$

Now, taking into account (6.3) and multiplying the equality (6.9) by $e^{k t}, 0<k<q \sigma$, we obtain

$$
\frac{d}{d t}\left(e^{k t} \int_{\Omega}\left|\nabla v^{q / 2}\right|^{2}\right) \leqslant \frac{q}{2 d} \frac{d}{d t}\left(e^{k t} \int_{\Omega} m v^{q}\right)+\frac{q^{2}}{2 d} e^{k t} \int_{\Omega} m^{2} v^{q}+\frac{\gamma q}{2 d} e^{k t} \int_{\Omega} m v^{q} .
$$

Integrating the last inequality on $(0, t)$ we have

$$
e^{k t} \int_{\Omega}\left|\nabla v^{q / 2}\right|^{2} \leqslant \int_{\Omega}\left|\nabla v_{0}^{q / 2}\right|^{2}+\frac{q}{2 d} e^{k t} \int_{\Omega} m v^{q}+\frac{q^{2}}{2 d} \int_{0}^{t} e^{k s} \int_{\Omega} m^{2} v^{q}+\frac{\gamma q}{2 d} \int_{0}^{t} e^{k s} \int_{\Omega} m v^{q} .
$$

Since $v(x, t)=v_{0} e^{-\int_{0}^{t} m}$ and $m(x, t) \geqslant \sigma>0$,

$$
v(x, t) \leqslant\left\|v_{0}\right\|_{L^{\infty}(\Omega)} e^{-\sigma t} .
$$

Introducing the estimate (6.12) in (6.11) and taking into account (4.3) and (4.7), we get

$$
\begin{aligned}
& \int_{\Omega}\left|\nabla v^{q / 2}(t)\right|^{2} \leqslant e^{-k t} \int_{\Omega}\left|\nabla v_{0}^{q / 2}\right|^{2}+ \\
& +\frac{q}{2 d}\left\|v_{0}\right\|_{L^{\infty}(\Omega)}^{q} \max \left\{\|m\|_{L^{1}(\Omega)}, C_{10}\right\}\left(e^{(k-\sigma q) t}+q \int_{0}^{t} e^{(k-\sigma q) s}+\gamma \int_{0}^{t} e^{(k-\sigma q) s}\right) e^{-k t} \leqslant \\
& \leqslant\left[\int_{\Omega}\left|\nabla v_{0}^{q / 2}\right|^{2}+\frac{q}{2 d} C_{32}\left\|v_{0}\right\|_{L^{\infty}(\Omega)}^{q}\left(1+\frac{q+\gamma}{\sigma q-k}\right)\right] e^{-k t},
\end{aligned}
$$


where

$$
C_{32}=\max \left\{C_{10},\left\|m_{0}\right\|_{L^{1}(\Omega)}+\left(L_{g}\left\|v_{0}\right\|_{L^{\infty}(\Omega)}+g(0)\right) \max \left\{|\Omega|,\left\|u_{0}\right\|_{L^{1}(\Omega)}\right\}\right\} .
$$

Finally, from (6.13) and denoting

$$
C_{33}=\left[\int_{\Omega}\left|\nabla v_{0}^{q / 2}\right|^{2}+\frac{q}{2 d} C_{32}\left\|v_{0}\right\|_{L^{\infty}(\Omega)}^{q}\left(1+\frac{q+\gamma}{\sigma q-k}\right)\right]
$$

we conclude the proof.

Theorem 6.3 If the hypotheses of Lemma 6.2 are satisfied, then

$$
\begin{aligned}
& \|u(\cdot, t)-\bar{u}\|_{L^{p}(\Omega)} \leqslant C_{36} e^{-C_{37} t}, \\
& \|v(\cdot, t)\|_{L^{\infty}(\Omega)} \leqslant C_{38} e^{-\sigma t}, \\
& \left\|m(\cdot, t)-\bar{u} \gamma^{-1} g(0)\right\|_{L^{p}(\Omega)} \leqslant C_{39} e^{-C_{40} t},
\end{aligned}
$$

where $C_{36}, C_{37}, C_{39}, C_{40}$ are positive constants independent on $t, C_{38}=\left\|v_{0}\right\|_{L^{\infty}(\Omega)}$ and

$$
\bar{u}= \begin{cases}\frac{1}{|\Omega|} \int_{\Omega} u_{0}, & \mu=0 \\ 1, & \mu>0\end{cases}
$$

Proof. Let $\alpha$ be a positive constant to be chosen later. By multiplying the equation (1.6) with $(u-\alpha)^{2 p+1}, p \geqslant 0$ and integrating in space we get

$$
\begin{aligned}
& \frac{d}{d t} \int_{\Omega}(u-\alpha)^{2 p+2} \leqslant-(p+1)(2 p+1) \int_{\Omega}(u-\alpha)^{2 p}|\nabla u|^{2}+ \\
& +2^{2 p}(p+1)(2 p+1) C_{25} \max \left\{C_{25}, \alpha^{2 p}\right\}\left(L_{\chi}\left\|v_{0}\right\|_{L^{\infty}(\Omega)}^{2}+\chi(0)\right)^{2} \int_{\Omega}|\nabla v|^{2}+ \\
& +2 \mu(p+1) \int_{\Omega} u(1-u)(u-\alpha)^{2 p+1}-2 \mu(p+1) \int_{\Omega} u^{2} v(u-\alpha)^{2 p}+2 \mu \alpha(p+1) \int_{\Omega} u v(u-\alpha)^{2 p} .
\end{aligned}
$$

In the case $\mu=0$ we consider $\alpha=\bar{u}=\frac{1}{\Omega \mid} \int_{\Omega} u_{0}$. Using the Poincaré inequality we obtain from (6.18)

$$
\frac{d}{d t} \int_{\Omega}(u-\bar{u})^{2}+C_{34} \int_{\Omega}(u-\bar{u})^{2} \leqslant\|u\|_{L^{\infty}\left(0, t ; L^{\infty}(\Omega)\right)}^{2}\left(L_{\chi}\left\|v_{0}\right\|_{L^{\infty}(\Omega)}^{2}+\chi(0)\right)^{2} \int_{\Omega}|\nabla v|^{2} .
$$

Using Lemma 6.2 for $q=1, k \neq C_{34}$ and applying the Gronwall inequality in the last estimate we have

$$
\|u(t)-\bar{u}\|_{L^{2}(\Omega)}^{2} \leqslant\left[\left\|u_{0}-\bar{u}\right\|_{L^{2}(\Omega)}^{2}+C_{25}^{2}\left(L_{\chi}\left\|v_{0}\right\|_{L^{\infty}(\Omega)}^{2}+\chi(0)\right)^{2} \frac{C_{33}}{\left|k-C_{34}\right|}\right] e^{-\min \left\{k, C_{34}\right\} t} .
$$

Moreover, for $p \geqslant 2$ it follows that

$$
\begin{aligned}
\|u(t)-\bar{u}\|_{L^{p}(\Omega)}^{p} & \leqslant 2^{p-2} \max \left\{\bar{u}^{p-2}, C_{25}^{p-2}\right\}\left[\left\|u_{0}-\bar{u}\right\|_{L^{2}(\Omega)}^{2}+\right. \\
& \left.+C_{25}^{2}\left(L_{\chi}\left\|v_{0}\right\|_{L^{\infty}(\Omega)}^{2}+\chi(0)\right)^{2} \frac{C_{33}}{\left|k-C_{34}\right|}\right] e^{-\min \left\{k, C_{34}\right\} t} .
\end{aligned}
$$


In the case $\mu>0$ we consider $\alpha=1$ and taking into account Lemma 6.1 and Lemma 6.2 we obtain from (6.18)

$$
\begin{aligned}
& \frac{d}{d t} \int_{\Omega}(u-1)^{2 p+2}+C_{35} \int_{\Omega}(u-1)^{2 p+2} \leqslant \\
& \leqslant 2^{2 p} C_{25} \max \left\{1, C_{25}^{2 p}\right\}\left[(p+1)(2 p+1) C_{25}\left(L_{\chi}\left\|v_{0}\right\|_{L^{\infty}(\Omega)}^{2}+\chi(0)\right)^{2} \int_{\Omega}|\nabla v|^{2}+2 \mu|\Omega|\left\|v_{0}\right\|_{L^{\infty}(\Omega)} e^{-\delta t}\right]
\end{aligned}
$$

where

$$
C_{35}=2 \mu(p+1) \min \{1, a\} e^{\int_{0}^{v_{0}} \chi(s) d s} .
$$

Applying Gronwall's inequality, using the estimate (6.6) and choosing $k \neq C_{35}$ we obtain

$$
\begin{aligned}
& \|u(t)-1\|_{L^{2 p+2}(\Omega)}^{2 p+2} \leqslant\left\{\left\|u_{0}-1\right\|_{L^{2 p+2}(\Omega)}^{2 p+2}+2^{2 p} C_{25} \max \left\{1, C_{25}^{2 p}\right\}\left[2 \mu|\Omega|\left\|v_{0}\right\|_{L^{\infty}(\Omega)}+\right.\right. \\
& \left.\left.+(p+1)(2 p+1) C_{25}\left(L_{\chi}\left\|v_{0}\right\|_{L^{\infty}(\Omega)}^{2}+\chi(0)\right)^{2} \frac{C_{33}}{\left|k-C_{35}\right|}\right]\right\} e^{-\min \left\{k, C_{35}\right\} t} .
\end{aligned}
$$

Taking into account (6.20) and (6.21) we conclude the estimate (6.14).

By multiplying the equation (1.6) with $\left(m-\bar{u} \gamma^{-1} g(0)\right)^{2 p+1}, p \geqslant 0$ and integrating in space we get

$$
\begin{aligned}
& \frac{d}{d t} \int_{\Omega}\left(m-\bar{u} \gamma^{-1} g(0)\right)^{2 p+2}=-2 d(p+1)^{2} \int_{\Omega}\left(m-\bar{u} \gamma^{-1} g(0)\right)^{2 p}|\nabla m|^{2}- \\
& -2 \gamma(p+1) \int_{\Omega}\left(m-\bar{u} \gamma^{-1} g(0)\right)^{2 p+2}+2(p+1) \int_{\Omega}\left(m-\bar{u} \gamma^{-1} g(0)\right)^{2 p+1}(u g(v)-\bar{u} g(0)) \leqslant \\
& \leqslant-2 d(p+1)^{2} \int_{\Omega}\left(m-\bar{u} \gamma^{-1} g(0)\right)^{2 p}|\nabla m|^{2}-\gamma(p+1) \int_{\Omega}\left(m-\bar{u} \gamma^{-1} g(0)\right)^{2 p+2}+ \\
& +\frac{(2 p+1)^{2 p+1}}{\gamma^{2 p+1}(p+1)^{4 p+3}} \int_{\Omega} \max \left\{L_{g} u^{2 p+2} v^{2 p+2}, g^{2 p+2}(0)|u-\bar{u}|^{2 p+2}\right\} \leqslant \\
& \leqslant-\gamma(p+1) \int_{\Omega}\left(m-\bar{u} \gamma^{-1} g(0)\right)^{2 p+2}+ \\
& +\frac{(2 p+1)^{2 p+1}}{\gamma^{2 p+1}(p+1)^{4 p+3}} \max \left\{L_{g}|\Omega|\left(C_{17}\|v(\cdot, t)\|_{L^{\infty}(\Omega)}\right)^{2 p+2}, g^{2 p+2}(0)\|u(\cdot, t)-\bar{u}\|_{L^{2 p+2}}^{2 p+2}\right\} .
\end{aligned}
$$

Using the estimates (6.14), (6.15) and the Gronwall lemma, when $\gamma \neq \min \left\{C_{37}, 2 \sigma\right\}$ we obtain

$$
\begin{aligned}
& \left\|m-\bar{u} \gamma^{-1} g(0)\right\|_{L^{2 p+2}}^{2 p+2} \leqslant\left[\left\|m_{0}-\bar{u} \gamma^{-1} g(0)\right\|_{L^{2 p+2}}^{2 p+2}+\right. \\
& \left.+\frac{(2 p+1)^{2 p+1} \max \left\{L_{g}|\Omega|\left(C_{17} C_{38}\right)^{2 p+2}, g^{2 p+2}(0) C_{36}\right\}}{\gamma^{2 p+1}(p+1)^{4 p+4}\left|\gamma-\min \left\{C_{37}, 2 \sigma\right\}\right|}\right] e^{-\min \left\{C_{37},(2 p+2) \sigma, \gamma(p+1)\right\} t}
\end{aligned}
$$

and we conclude the estimate (6.16).

In the next theorem we shall prove that starting with initial data in suitable spaces we obtain stronger convergences. 
Theorem 6.4 Let $\left(u_{0}, v_{0}, m_{0}\right) \in L^{\infty}(\Omega) \times W^{1, \infty}(\Omega) \times W^{1, p}(\Omega), p>3$. Under the hypotheses of Lemma 6.1, we have

$$
\begin{aligned}
& \|v(\cdot, t)\|_{W^{1, \infty}} \leqslant C_{41}(1+t) e^{-\sigma t}, \\
& \left\|m(\cdot, t)-\bar{u} \gamma^{-1} g(0)\right\|_{X_{p}^{\theta}} \leqslant C_{42}(1+t) t^{-\theta} e^{-C_{43} t},
\end{aligned}
$$

for all $t \in(0, t)$. Moreover, if the hypotheses of Lemma 4.11 are satisfied, then

$$
\|u(\cdot, t)-\bar{u}\|_{W^{1, \infty}} \leqslant C_{44} e^{-\frac{1}{2} C_{43} t}
$$

for $t$ sufficiently large. The constants $C_{41}, C_{42}, C_{43}, C_{44}$ are positive and independent on $t$, and $\bar{u}$ is given by 6.17).

Proof. Let $\theta \in\left(\frac{3+p}{2 p}, 1\right)$. From the representation formula (4.6) and taking into account (2.4), (4.14), (6.14), 6.15) we obtain

$$
\begin{aligned}
& \left\|m(\cdot, t)-\bar{u} \gamma^{-1} g(0)\right\|_{X_{p}^{\theta}} \leqslant\left\|e^{-t A_{2}}\left[m_{0}-\bar{u} \gamma^{-1} g(0)\right]\right\|_{X_{p}^{\theta}}+\int_{0}^{t}\left\|A_{2}^{\theta} e^{-(t-s) A_{2}}[u g(v)-\bar{u} g(0)]\right\|_{L^{p}(\Omega)} d s \leqslant \\
& \leqslant C(\theta) t^{-\theta} e^{-\delta t}\left(\left\|m_{0}\right\|_{L^{p}(\Omega)}+\left\|\bar{u} \gamma^{-1} g(0)\right\|_{L^{p}(\Omega)}\right)+ \\
& +C(\theta, p) \int_{0}^{t}(t-s)^{-\theta} e^{-\delta(t-s)}\left[\|u g(v)-u g(0)\|_{L^{p}(\Omega)}+\|u g(0)-\bar{u} g(0)\|_{L^{p}(\Omega)}\right] d s \leqslant \\
& \leqslant C(\theta) t^{-\theta} e^{-\delta t}\left(\left\|m_{0}\right\|_{L^{p}(\Omega)}+\bar{u} \gamma^{-1} g(0)|\Omega|\right)+ \\
& +C(\theta, p) L_{g} C_{17} C_{38} \int_{0}^{t}(t-s)^{-\theta} e^{-\delta(t-s)} e^{-\sigma s} d s+C(\theta, p) C_{36} g(0) \int_{0}^{t}(t-s)^{-\theta} e^{-\delta(t-s)} e^{-C_{37} s} d s \leqslant \\
& \leqslant \max \left\{C(\theta)\left(\left\|m_{0}\right\|_{L^{p}(\Omega)}+\bar{u} \gamma^{-1} g(0)|\Omega|\right), \frac{C(\theta, p)}{1-\theta}\left(L_{g} C_{17} C_{38}+g(0) C_{36}\right)\right\}(1+t) t^{-\theta} e^{-\min \left\{\sigma, \delta, C_{37}\right\} t .}
\end{aligned}
$$

Denoting

$$
\begin{aligned}
& C_{42}=\max \left\{C(\theta)\left(\left\|m_{0}\right\|_{L^{p}(\Omega)}+\bar{u} \gamma^{-1} g(0)|\Omega|\right), \frac{C(\theta, p)}{1-\theta}\left(L_{g} C_{17} C_{38}+g(0) C_{36}\right)\right\}, \\
& C_{43}=\min \left\{\sigma, \delta, C_{37}\right\}
\end{aligned}
$$

the last inequality implies the estimate (6.23).

Taking into account (4.27), the estimate (6.24) and the embedding (2.3), it follows that

$$
\begin{aligned}
\|\nabla v\|_{L^{\infty}} & \leqslant e^{-\sigma t}\left(\left\|\nabla v_{0}\right\|_{L^{\infty}}+\left\|v_{0}\right\|_{L^{\infty}} \int_{0}^{t}\left\|m-\bar{u} \gamma^{-1} g(0)\right\|_{W^{1, \infty}} d s\right) \leqslant \\
& \leqslant e^{-\sigma t}\left(\left\|\nabla v_{0}\right\|_{L^{\infty}}+C_{42}\left\|v_{0}\right\|_{L^{\infty}} \int_{0}^{t}(1+s) s^{-\theta} e^{-C_{43} s} d s\right) \leqslant \\
& \leqslant 2 \max \left\{1, C_{42} C_{43}^{\theta-1} \Gamma(1-\theta)\right\}\left\|v_{0}\right\|_{W^{1, \infty}}(1+t) e^{-\sigma t} .
\end{aligned}
$$

The last inequality together with (6.15) imply (6.22) where

$$
C_{41}=\left(1+2 \max \left\{1, C_{42} C_{43}^{\theta-1} \Gamma(1-\theta)\right\}\right)\left\|v_{0}\right\|_{W^{1, \infty}} .
$$

We start now to prove (6.24). Let $t_{0}>0$ sufficiently large. Taking into account the embedding (2.3), we obtain from (6.23)

$$
\|\nabla m\|_{L^{\infty}(\Omega)} \leqslant C_{45} e^{-\frac{1}{2} C_{43} t}
$$


for all $t>t_{0}$. If we take also into account (4.27), the last inequality implies

$$
\begin{aligned}
\|\nabla v\|_{L^{\infty}(\Omega)} & \leqslant e^{-\sigma t}\left(\left\|\nabla v_{0}\right\|_{L^{\infty}(\Omega)}+C_{45}\left\|v_{0}\right\|_{L^{\infty}(\Omega)} \int_{0}^{t} e^{-\frac{1}{2} C_{43} t}\right) \leqslant \\
& \leqslant \max \left\{1, \frac{2 C_{45}}{C_{43}}\right\}\left\|v_{0}\right\|_{W^{1, \infty}(\Omega)} e^{-\sigma t}
\end{aligned}
$$

for $t$ sufficiently large. From the representation formula

$$
w(x, t)=e^{-\left(t-t_{0}\right) A_{1}} w\left(t_{0}\right)+\int_{t_{0}}^{t} e^{-(t-s) A_{1}} G_{1}(w, v, m)(s) d s
$$

and taking into account (4.8), (4.15) and (6.15), we obtain

$$
\begin{aligned}
& \|w(\cdot, t)-\bar{u}\|_{X_{p}^{\theta}} \leqslant\left\|e^{-\left(t-t_{0}\right) A_{1}}\left(w\left(t_{0}\right)-\bar{u}\right)\right\|_{X_{p}^{\theta}}+ \\
& +\int_{t_{0}}^{t}\left\|A_{1}^{\theta} e^{-(t-s) A_{1}}\left[\chi(v) \nabla v \cdot \nabla w+(\mu+1) w-\mu w\left(w z^{-1}+v\right)+\chi(v) w v m-\bar{u}\right]\right\|_{L^{p}(\Omega)} d s \leqslant \\
& \leqslant C(\theta)\left(t-t_{0}\right)^{-\theta} e^{-\delta\left(t-t_{0}\right)}\left\|w\left(t_{0}\right)-\bar{u}\right\|_{L^{p}(\Omega)}+ \\
& +C(\theta, p)\left(L_{\chi}\left\|v_{0}\right\|_{L^{\infty}(\Omega)}+\chi(0)\right) \int_{t_{0}}^{t}(t-s)^{-\theta} e^{-\delta(t-s)}\|\nabla v\|_{L^{\infty}(\Omega)}\|\nabla w\|_{L^{p}(\Omega)} d s+ \\
& +\mu C_{18} C(\theta, p) \int_{t_{0}}^{t}(t-s)^{-\theta} e^{-\delta(t-s)}\|u-1\|_{L^{p}(\Omega)}+C(\theta, p) \int_{t_{0}}^{t}(t-s)^{-\theta} e^{-\delta(t-s)}\|w-\bar{u}\|_{L^{p}(\Omega)}+ \\
& +\mu C_{11} C_{38} C(\theta, p) \int_{t_{0}}^{t}(t-s)^{-\theta} e^{-\delta(t-s)} e^{-\sigma s} d s+ \\
& +C_{11} C_{38} C(\theta, p)\left(L_{\chi}\left\|v_{0}\right\|_{L^{\infty}(\Omega)}+\chi(0)\right) \int_{t_{0}}^{t}(t-s)^{-\theta} e^{-\delta(t-s)} e^{-\sigma s}\|m\|_{L^{\infty}(\Omega)} \cdot
\end{aligned}
$$

Let us observe that

$$
\|w-u\|_{L^{p}(\Omega)}^{p} \leqslant\|u\|_{L^{\infty}(\Omega)}^{p}\|z-1\|_{L^{p}(\Omega)}^{p} \leqslant C_{46}\|u\|_{L^{\infty}(\Omega)}^{p} \int_{\Omega}\left|\int_{0}^{v} \chi(s) d s\right|^{p} \leqslant C_{47}^{p} e^{-\sigma p t},
$$

where

$$
C_{47}=2 C_{46}^{1 / p} C_{25} C_{38}|\Omega|^{1 / p} \max \left\{C_{38}\left(\frac{L_{\chi}}{2}\right), \chi(0)\right\} .
$$

From the above inequality, using (6.14), it follows also that

$$
\|w-\bar{u}\|_{L^{p}(\Omega)} \leqslant \max \left\{C_{36}, C_{47}\right\} e^{-\min \left\{\sigma, C_{37}\right\} t} .
$$

Using the estimates (4.25), (6.14), (6.25), 6.15) and 6.27), we obtain from (6.26)

$$
\begin{aligned}
\|w(\cdot, t)-\bar{u}\|_{X_{p}^{\theta}} & \leqslant C_{48}\left(t-t_{0}\right)^{1-\theta} e^{-\min \left\{\delta, \sigma, C_{37}\right\} t}+C_{49}\left(t-t_{0}\right)^{-\theta} e^{-\delta t}+ \\
& +C_{50} \int_{t_{0}}^{t}(t-s)^{-\theta} e^{-\delta(t-s)} e^{-\sigma s}\|\nabla w\|_{L^{p}(\Omega)} d s
\end{aligned}
$$


where

$$
\begin{aligned}
C_{48} & =\frac{C(\theta, p)}{1-\theta}\left\{\left[\max \left\{C_{36}, C_{47}\right\}+C_{11} C_{26} C_{38}\left(L_{\chi}\left\|v_{0}\right\|_{L^{\infty}(\Omega)}+\chi(0)\right)\right]+\mu\left(C_{18} C_{36}+C_{11} C_{38}\right)\right\} \\
C_{49} & =C(\theta) e^{\delta t_{0}}\left(C_{11}+\|\bar{u}\|_{L^{p}(\Omega)}\right) \\
C_{50} & =C(\theta, p)\left(L_{\chi}\left\|v_{0}\right\|_{L^{\infty}(\Omega)}+\chi(0)\right) \max \left\{1, \frac{2 C_{42}}{C_{43}}\right\}\left\|v_{0}\right\|_{W^{1, \infty}(\Omega)} .
\end{aligned}
$$

Taking into account the embedding (2.2) and applying the Gronwall lemma we obtain from the last inequality

$$
\begin{aligned}
& \|w(\cdot, t)-\bar{u}\|_{X_{p}^{\theta}} \leqslant C_{48}\left(t-t_{0}\right)^{1-\theta} e^{-\min \left\{\delta, \sigma, C_{37}\right\} t}+C_{49}\left(t-t_{0}\right)^{-\theta} e^{-\delta t}+ \\
& +C_{50} e^{C_{50} \delta^{\theta-1} \Gamma(1-\theta)}\left[C_{48} e^{-\min \left\{\delta, \sigma, C_{37}\right\} t} \int_{t_{0}}^{t}\left(s-t_{0}\right)^{1-\theta}(t-s)^{-\theta} d s+C_{49} e^{-\delta t} \int_{t_{0}}^{t}\left(s-t_{0}\right)^{-\theta}(t-s)^{-\theta} d s\right] \leqslant \\
& \leqslant C_{45}\left(t-t_{0}\right)^{1-\theta} e^{-\min \left\{\delta, \sigma, C_{37}\right\} t}+C_{49}\left(t-t_{0}\right)^{-\theta} e^{-\delta t}+ \\
& +C_{50} e^{C_{50} \delta^{\theta-1} \Gamma(1-\theta)} \frac{2^{2 \theta-2} \sqrt{\pi}\left(t-t_{0}\right)^{1-2 \theta} \Gamma(1-\theta)}{\Gamma\left(\frac{3}{2}-\theta\right)}\left(C_{48}\left(t-t_{0}\right)+2 C_{49}\right) e^{-\min \left\{\delta, \sigma, C_{37}\right\} t} \leqslant \\
& \leqslant\left(t-t_{0}\right)^{-\theta}\left(C_{48}\left(t-t_{0}\right)+2 C_{49}\right)\left(1+2^{2 \theta-2} C_{50} \frac{\sqrt{\pi}\left(t-t_{0}\right)^{1-\theta} \Gamma(1-\theta)}{\Gamma\left(\frac{3}{2}-\theta\right)} e^{C_{50} \delta^{\theta-1} \Gamma(1-\theta)}\right) e^{-\min \left\{\delta, \sigma, C_{37}\right\} t} .
\end{aligned}
$$

Let us observe that

$$
\|u(\cdot, t)-\bar{u}\|_{W^{1, \infty}} \leqslant\left\|z^{-1}\right\|_{W^{1, \infty}} \cdot\|w-\bar{u}\|_{W^{1, \infty}}+\|\bar{u}\|_{L^{\infty}}\left\|z^{-1}-1\right\|_{W^{1, \infty}} .
$$

Using (6.25) we obtain

$$
\left\|z^{-1}\right\|_{W^{1, \infty}} \leqslant C_{12}\left[1+C_{12}\left(L_{\chi}\left\|v_{0}\right\|_{L^{\infty}(\Omega)}+\chi(0)\right) \max \left\{1, \frac{2 C_{45}}{C_{43}}\right\}\left\|v_{0}\right\|_{W^{1, \infty}(\Omega)}\right] e^{-\sigma t} .
$$

Taking into account (6.15) and (6.25), we estimate

$$
\begin{aligned}
& \left\|z^{-1}-1\right\|_{W^{1, \infty}} \leqslant C_{46}\left\|\int_{0}^{v} \chi(s) d s\right\|_{L^{\infty}}+C_{12}\left(L_{\chi}\left\|v_{0}\right\|_{L^{\infty}(\Omega)}+\chi(0)\right) \max \left\{1, \frac{2 C_{45}}{C_{43}}\right\}\left\|v_{0}\right\|_{W^{1, \infty}(\Omega)} e^{-\sigma t} \leqslant \\
& \leqslant\left[C_{46} C_{38}\left(\frac{L_{\chi}}{2} C_{38}+\chi(0)\right)+C_{12}\left(L_{\chi}\left\|v_{0}\right\|_{L^{\infty}(\Omega)}+\chi(0)\right) \max \left\{1, \frac{2 C_{45}}{C_{43}}\right\}\left\|v_{0}\right\|_{W^{1, \infty}(\Omega)}\right] e^{-\sigma t}
\end{aligned}
$$

From (6.29), using the embedding (2.2) and the estimates (6.28), (6.30), (6.31), we obtain

$$
\begin{aligned}
& \|u(t)-\bar{u}\|_{W^{1, \infty}} \leqslant C_{51}\|w-\bar{u}\|_{W^{1, \infty}}+C_{52} \bar{u} e^{-\sigma t} \leqslant \\
& \leqslant\left[C_{51}\left(t-t_{0}\right)^{-\theta}\left(C_{48}\left(t-t_{0}\right)+2 C_{49}\right)\left(1+2^{2 \theta-2} C_{50} \frac{\sqrt{\pi}\left(t-t_{0}\right)^{1-\theta} \Gamma(1-\theta)}{\Gamma\left(\frac{3}{2}-\theta\right)} e^{C_{50} \delta^{\theta-1} \Gamma(1-\theta)}\right)+C_{52} \bar{u}\right] e^{-\min \left\{\delta, \sigma, C_{37}\right\} t}
\end{aligned}
$$

where

$$
\begin{aligned}
C_{51} & =C_{12}\left[1+C_{12}\left(L_{\chi}\left\|v_{0}\right\|_{L^{\infty}(\Omega)}+\chi(0)\right) \max \left\{1, \frac{2 C_{45}}{C_{43}}\right\}\left\|v_{0}\right\|_{W^{1, \infty}(\Omega)}\right] \\
C_{52} & =\left[C_{46} C_{38}\left(\frac{L_{\chi}}{2} C_{38}+\chi(0)\right)+C_{12}\left(L_{\chi}\left\|v_{0}\right\|_{L^{\infty}(\Omega)}+\chi(0)\right) \max \left\{1, \frac{2 C_{45}}{C_{43}}\right\}\left\|v_{0}\right\|_{W^{1, \infty}(\Omega)}\right]
\end{aligned}
$$

The last inequality implies (6.24) for $t$ sufficiently large.

In what follows we investigate the case when $g(0)=0$ and we start with an auxiliary lemma. 
Lemma 6.5 Let $f \in C^{1}(0,+\infty)$ satisfying

$$
\int_{0}^{\infty}|f(s)| d s \leqslant C, \quad \int_{0}^{\infty}\left|f^{\prime}(s)\right| d s \leqslant C
$$

then

$$
\lim _{t \rightarrow \infty} f(t)=0 .
$$

Proof. Assume that $\lim _{t \rightarrow \infty} f(t) \neq 0$, then there exists a sequence $\left\{t_{n}\right\}_{n \in \mathbb{N}}, t_{n} \rightarrow \infty$ such that

$$
\left|f\left(t_{n}\right)\right|>C>0, \quad \forall n \geqslant n_{0} .
$$

We can assume that $t_{n+1}>t_{n}+1$ (otherwise we take a subsequence). Let $0<\tau<1$, then for all $n \geqslant n_{0}$

$$
|| f\left(t_{n}+\tau\right)|-| f\left(t_{n}\right)|| \leqslant\left|f\left(t_{n}+\tau\right)-f\left(t_{n}\right)\right|=\left|\int_{t_{n}}^{t_{n}+\tau} f^{\prime}(s) d s\right| \leqslant \int_{t_{n}}^{t_{n}+\tau}\left|f^{\prime}(s)\right| d s \leqslant \int_{t_{n}}^{t_{n}+1}\left|f^{\prime}(s)\right| d s .
$$

But

$$
\lim _{n \rightarrow \infty} \int_{t_{n}}^{t_{n}+1}\left|f^{\prime}(s)\right| d s=0
$$

(otherwise, on a subsequence, $\int_{t_{n}}^{t_{n}+1}\left|f^{\prime}(s)\right| d s \geqslant C$ which implies $\left.\int_{0}^{\infty}\left|f^{\prime}(s)\right| d s \geqslant \sum_{n} \int_{t_{n}}^{t_{n}+1}\left|f^{\prime}(s)\right| d s \rightarrow \infty\right)$.

Then for $n \geqslant n_{1} \geqslant n_{0}$ we have

$$
\int_{t_{n}}^{t_{n}+1}\left|f^{\prime}(s)\right| d s<\frac{C}{2}
$$

Therefore

$$
\left|f\left(t_{n}+\tau\right)\right|>\frac{C}{2}
$$

which implies

$$
\int_{t_{n}}^{t_{n}+1}|f(s)| d s \geqslant \frac{C}{2}
$$

which contradicts the hypothesis.

Theorem 6.6 Let $\mu>0$ and $\left(u_{0}, v_{0}, m_{0}\right) \in L^{1}(\Omega) \times\left(W^{1,2}(\Omega) \cap L^{\infty}(\Omega)\right) \times W^{1, p}(\Omega), p \geqslant \frac{6}{5}$. Let $g$ be a function satisfying the hypotheses $(H 2)$ and $(H 3)$ and $g(0)=0$. Then

$$
\begin{aligned}
& \lim _{t \rightarrow \infty}\|m(\cdot, t)\|_{L^{2}(\Omega)}=0, \\
& \lim _{t \rightarrow \infty}\left\|g(v(\cdot, t)) v^{q}(\cdot, t)\right\|_{L^{1}(\Omega)}=0, \quad q \geqslant 1 .
\end{aligned}
$$

Moreover, if $g(v) \geqslant C_{53} v$ for all $v \geqslant 0$, where $C_{53}$ is a positive constant independent on $t$, then

$$
\lim _{t \rightarrow \infty}\|u(\cdot, t)-\bar{u}\|_{L^{2}(\Omega)}=0
$$

where $\bar{u}$ is given by 6.17). 
Proof. On multiplying the equation (1.8) by $m$, integrating over $\Omega$ and taking into account the estimates (3.8), (4.15) and the hypothesis $\left(H_{3}\right)$, we obtain

$$
\frac{1}{2} \frac{d}{d t} \int_{\Omega} m^{2}+d \int_{\Omega}|\nabla m|^{2}+\gamma \int_{\Omega} m^{2}=-L_{g} \int_{\Omega} u v_{t} .
$$

Integrating in time the last equality we get

$$
\frac{1}{2} \int_{\Omega} m^{2}+d \int_{0}^{t} \int_{\Omega}|\nabla m|^{2}+\gamma \int_{0}^{t} \int_{\Omega} m^{2} \leqslant C_{25} L_{g} \int_{\Omega} v_{0}+\frac{1}{2} \int_{\Omega} m_{0}^{2} .
$$

Therefore we have

$$
\begin{gathered}
\int_{0}^{t} \int_{\Omega}|\nabla m|^{2} \leqslant C, \\
\int_{0}^{t} \int_{\Omega} m^{2} \leqslant C .
\end{gathered}
$$

From (6.35) we deduce

$$
\left|\frac{d}{d t} \int_{\Omega} m^{2}\right| \leqslant-2 C_{12} C_{18} L_{g} \int_{\Omega} v_{t}+2 d \int_{\Omega}|\nabla m|^{2}+2 \gamma \int_{\Omega} m^{2}
$$

and integrating in time we obtain

$$
\int_{0}^{t}\left|\frac{d}{d t} \int_{\Omega} m^{2}\right| \leqslant-2 C_{12} C_{18} L_{g} \int_{0}^{t} \int_{\Omega} v_{t}+2 d \int_{0}^{t} \int_{\Omega}|\nabla m|^{2}+2 \gamma \int_{0}^{t} \int_{\Omega} m^{2} .
$$

The last inequality together with (6.36), (6.37) and Lemma 6.5 imply (6.32).

In order to deal with the convergence of $v$, we integrate in time the estimate (6.9) and we obtain

$$
\begin{aligned}
& \int_{\Omega}\left|\nabla v^{q / 2}\right|^{2}+q \int_{0}^{t} \int_{\Omega} m\left|\nabla v^{q / 2}\right|^{2}+\frac{q}{2 d} \int_{0}^{t} \int_{\Omega} u g(v) v^{q} \leqslant \\
& \leqslant \int_{\Omega}\left|\nabla v_{0}^{q / 2}\right|^{2}+\frac{q}{2 d} \int_{\Omega} m v^{q}+\frac{q^{2}}{2 d} \int_{0}^{t} \int_{\Omega} m^{2} v^{q}+\frac{\gamma q}{2 d} \int_{0}^{t} \int_{\Omega} m v^{q} .
\end{aligned}
$$

The last inequality, taking into account also (6.1), implies

$$
\int_{0}^{t} \int_{\Omega} g(v) v^{q} \leqslant C_{54}
$$

On the other hand

$$
\begin{aligned}
\int_{0}^{t}\left|\frac{d}{d t} \int_{\Omega} g(v) v^{q}\right| & \leqslant-L_{g^{\prime}} \int_{0}^{t} \int_{\Omega} v^{q+1} v_{t}-\left|g^{\prime}(0)\right| \int_{0}^{t} \int_{\Omega} v^{q} v_{t}-q L_{g} \int_{0}^{t} \int_{\Omega} v^{q} v_{t} \leqslant \\
& \leqslant\left(L_{g^{\prime}}\left\|v_{0}\right\|_{L^{\infty}(\Omega)}+\left|g^{\prime}(0)\right|+q L_{g}\right)|\Omega|\left\|v_{0}\right\|_{L^{\infty}(\Omega)}^{q+1} \leqslant C .
\end{aligned}
$$


From Lemma 6.5, (6.39) and (6.40) we get (6.33).

In order to have the last estimate we multiply the equation (1.12) by $(w-a)$, where $a$ is a constant to be determined later and we obtain

$$
\begin{aligned}
\frac{1}{2} \frac{d}{d t} \int_{\Omega}\left[z^{-1}(w-a)^{2}\right] & =-\int_{\Omega} z^{-1}|\nabla w|^{2}+\mu \int_{\Omega} w z^{-1}(1-w)(w-a)+\mu \int_{\Omega} w^{2} z^{-1}\left(1-z^{-1}\right)(w-a)- \\
& -\mu \int_{\Omega} w z^{-1} v(w-a)+\int_{\Omega} \chi(v) w^{2} z^{-1} m v-a \int_{\Omega} \chi(v) w z^{-1} m v .
\end{aligned}
$$

We consider first the case $\mu=0$. Taking $a=\bar{u}=\frac{1}{|\Omega|} \int_{\Omega} u$, from the equation (1.13) and integrating in time the last inequality we get

$$
\begin{aligned}
& \int_{\Omega} z^{-1}(w-\bar{u})^{2}+2 \int_{0}^{t} \int_{\Omega} z^{-1}|\nabla w|^{2} \leqslant \int_{\Omega}\left[z_{0}^{-1}\left(w_{0}-\bar{u}\right)^{2}\right]+2 \int_{0}^{t} \int_{\Omega} w^{2} z^{-1} \chi(v) m v \leqslant \\
& \leqslant \int_{\Omega}\left[z_{0}^{-1}\left(w_{0}-\bar{u}\right)^{2}\right]+2 C_{12}|\Omega|\left(L_{\chi}\left\|v_{0}\right\|_{L^{\infty}(\Omega)}+\chi(0)\right)\left\|v_{0}\right\|_{L^{\infty}(\Omega)}\|w\|_{L^{\infty}\left(0, t ; L^{\infty}(\Omega)\right)}^{2} .
\end{aligned}
$$

Therefore we have

$$
\int_{0}^{t} \int_{\Omega}|\nabla w|^{2} \leqslant \int_{0}^{t} \int_{\Omega} z^{-1}|\nabla w|^{2} \leqslant C_{55}
$$

From (6.41) we deduce

$$
\left|\frac{d}{d t} \int_{\Omega}\left[z^{-1}(w-\bar{u})^{2}\right]\right| \leqslant 2 \int_{\Omega} z^{-1}|\nabla w|^{2}-2 \int_{\Omega} \chi(v) w^{2} z^{-1} v_{t}-2 \bar{u} \int_{\Omega} \chi(v) w z^{-1} v_{t} .
$$

Integrating in time the last inequality and taking into account (4.1), (4.15) and (6.42) we obtain

$$
\int_{0}^{t}\left|\frac{d}{d t} \int_{\Omega}\left[z^{-1}(w-\bar{u})^{2}\right]\right| \leqslant 2 \int_{0}^{t} \int_{\Omega} z^{-1}|\nabla w|^{2}-2 \int_{0}^{t} \int_{\Omega} \chi(v) w^{2} z^{-1} v_{t}-2 \bar{u} \int_{0}^{t} \int_{\Omega} \chi(v) w z^{-1} v_{t} \leqslant C_{56}
$$

where

$$
C_{56}=2 C_{55}+2 C_{12} C_{18}|\Omega|^{2}\left(\max \left\{1,\left\|u_{0}\right\|_{L^{\infty}(\Omega)}\right\}+C_{18}\right)\left(L_{\chi}\left\|v_{0}\right\|_{L^{\infty}(\Omega)}+\chi(0)\right)\left\|v_{0}\right\|_{L^{\infty}(\Omega)} .
$$

Let us remark that we have the following estimate by using Poincaré inequality and (6.42)

$$
\begin{aligned}
\int_{0}^{t} \int_{\Omega} z^{-1}(w-\bar{u})^{2} & \leqslant 2 C_{12} \int_{0}^{t} \int_{\Omega}(w-\bar{w})^{2}+2 C_{12} \int_{0}^{t} \int_{\Omega}(\bar{w}-\bar{u})^{2} \leqslant \\
& \leqslant 2 C C_{12} \int_{0}^{t} \int_{\Omega}|\nabla w|^{2}+2 C_{12} \int_{0}^{t} \int_{\Omega}(\bar{w}-\bar{u})^{2} \leqslant C+2 C_{12} \int_{0}^{t} \int_{\Omega}(\bar{w}-\bar{u})^{2}
\end{aligned}
$$

where $\bar{w}=\frac{1}{|\Omega|} \int_{\Omega} w$. Then, in order to obtain the estimate (6.34) using Lemma 6.5 it is enough to prove that

$$
\int_{0}^{t} \int_{\Omega}(\bar{w}-\bar{u})^{2} \leqslant C
$$


Using (6.39) for $q=1$, an easy calculation shows us that

$$
\int_{0}^{t} \int_{\Omega}\left(1-z^{-1}\right)^{2} \leqslant C_{12} \max _{s \in\left(0,\left\|v_{0}\right\|_{L^{\infty}(\Omega)}\right.} \chi^{2}(s) \int_{0}^{t} \int_{\Omega} v^{2} \leqslant C_{12} C_{53} C_{54} \max _{s \in\left(0,\left\|v_{0}\right\|_{L^{\infty}(\Omega)}\right)} \chi^{2}(s) .
$$

Taking into account the last estimate we obtain

$$
\begin{aligned}
\int_{0}^{t} \int_{\Omega}(\bar{w}-\bar{u})^{2} & \leqslant \int_{0}^{t} \int_{\Omega} \frac{1}{|\Omega|^{2}} \int_{\Omega}(w-u)^{2} \leqslant \frac{1}{|\Omega|} C_{18}^{2} \int_{0}^{t} \int_{\Omega}\left(1-z^{-1}\right)^{2} \leqslant \\
& \leqslant \frac{1}{|\Omega|} C_{12} C_{18}^{2} C_{53} C_{54} \max _{s \in\left(0,\left\|v_{0}\right\|_{L}(\Omega)\right.} \chi^{2}(s)
\end{aligned}
$$

which implies (6.45). From (6.43), (6.44) and (6.47) the estimate (6.34) follows.

We consider now the case $\mu>0$. From (6.41) we obtain

$$
\begin{aligned}
& \frac{1}{2} \frac{d}{d t} \int_{\Omega}\left[z^{-1}(w-1)^{2}\right]=-\int_{\Omega} z^{-1}|\nabla w|^{2}-\mu \int_{\Omega} w z^{-1}(w-1)^{2}+\mu \int_{\Omega} w^{2} z^{-1}\left(1-z^{-1}\right)(w-1)- \\
& -\mu \int_{\Omega} w z^{-1} v(w-1)-\int_{\Omega} \chi(v) z^{-1} w^{2} v_{t} \leqslant \\
& \leqslant-\int_{\Omega} z^{-1}|\nabla w|^{2}-\frac{\mu}{2} \int_{\Omega} w z^{-1}(w-1)^{2}+\int_{\Omega} w^{3} z^{-1}\left(1-z^{-1}\right)^{2}+\int_{\Omega} w z^{-1} v^{2}-C_{57} \int_{\Omega} w^{2} v_{t},
\end{aligned}
$$

where

$$
C_{57}=e^{\frac{L_{\chi}}{2}\left\|v_{0}\right\|_{L^{\infty}(\Omega)}^{2}+\chi(0)\left\|v_{0}\right\|_{L^{\infty}(\Omega)}}\left(L_{\chi}\left\|v_{0}\right\|_{L^{\infty}(\Omega)}+\chi(0)\right) .
$$

From the above it follows that

$$
\begin{aligned}
& \frac{1}{2} \frac{d}{d t} \int_{\Omega}\left[z^{-1}(w-1)^{2}\right]+\frac{\mu}{2} \min \{1, a\} \int_{\Omega} z^{-1}(w-1)^{2}+\int_{\Omega} z^{-1}|\nabla w|^{2} \leqslant \\
& \leqslant \int_{\Omega} w^{3} z^{-1}\left(1-z^{-1}\right)^{2}+\int_{\Omega} w z^{-1} v^{2}-C_{57} \int_{\Omega} w^{2} v_{t} .
\end{aligned}
$$

Integrating the last inequality on $(0, t)$ we have

$$
\begin{aligned}
& \frac{1}{2} \int_{\Omega}\left[z^{-1}(w-1)^{2}\right]+\frac{\mu}{2} \min \{1, a\} \int_{0}^{t} \int_{\Omega} z^{-1}(w-1)^{2}+\int_{0}^{t} \int_{\Omega} z^{-1}|\nabla w|^{2} \leqslant \\
& \leqslant C_{12} C_{18}^{3} \int_{0}^{t} \int_{\Omega}\left(1-z^{-1}\right)^{2}+C_{12} C_{18} \int_{0}^{t} \int_{\Omega} v^{2}+C_{18}^{2} C_{57}|\Omega|\left\|v_{0}\right\|_{L^{\infty}(\Omega)}+\frac{1}{2} \int_{\Omega}\left(w_{0}-1\right)^{2} e^{\int_{0}} \chi(s) d s .
\end{aligned}
$$

Taking into account (6.46) and (6.39) with $q=1$, we obtain from (6.49)

$$
\frac{1}{2} \int_{\Omega}\left[z^{-1}(w-1)^{2}\right]+\frac{\mu}{2} \min \{1, a\} \int_{0}^{t} \int_{\Omega} z^{-1}(w-1)^{2}+\int_{0}^{t} \int_{\Omega} z^{-1}|\nabla w|^{2} \leqslant C_{58},
$$

where

$$
C_{58}=C_{12} C_{18} C_{54}\left(C_{12} C_{18} \max _{s \in\left(0,\left\|v_{0}\right\|_{L^{\infty}(\Omega)}\right)} \chi^{2}(s)+C_{53}^{-1}\right)+C_{57} C_{18}^{2}|\Omega|\left\|v_{0}\right\|_{L^{\infty}(\Omega)}+\frac{1}{2} \int_{\Omega}\left(w_{0}-1\right)^{2} e^{\int_{0}^{v_{0}} \chi(s) d s} .
$$


The inequality (6.50) implies

$$
\begin{gathered}
\int_{0}^{t} \int_{\Omega} z^{-1}(w-1)^{2} \leqslant C, \\
\int_{0}^{t} \int_{\Omega} z^{-1}|\nabla w|^{2} \leqslant C .
\end{gathered}
$$

From 6.48) we deduce that

$$
\begin{aligned}
\left|\frac{d}{d t} \int_{\Omega}\left[z^{-1}(w-1)^{2}\right]\right| & \leqslant 2 \int_{\Omega} z^{-1}|\nabla w|^{2}+2 \mu \int_{\Omega} w z^{-1}(w-1)^{2}+2 \mu \int_{\Omega} w^{2} z^{-1}\left|\left(1-z^{-1}\right)(w-1)\right|+ \\
& +2 \mu \int_{\Omega} w z^{-1} v|w-1|-2 \int_{\Omega} \chi(v) z^{-1} w^{2} v_{t}
\end{aligned}
$$

and integrating in time we have

$$
\begin{aligned}
& \int_{0}^{t}\left|\frac{d}{d t} \int_{\Omega}\left[z^{-1}(w-1)^{2}\right]\right| \leqslant 2 \int_{0}^{t} \int_{\Omega} z^{-1}|\nabla w|^{2}+2 \mu C_{18} \int_{0}^{t} \int_{\Omega} z^{-1}(w-1)^{2}+\mu C_{12} C_{18}^{2} \int_{0}^{t} \int_{\Omega}\left(1-z^{-1}\right)^{2}+ \\
& +\mu C_{18}^{2} \int_{0}^{t} \int_{\Omega} z^{-1}(w-1)^{2}+\mu C_{12} C_{18}^{2} \int_{0}^{t} \int_{\Omega} v^{2}+\mu \int_{0} \int_{\Omega} z^{-1}(w-1)^{2}- \\
& -2 C_{12} C_{18}^{2}\left(L_{\chi}\left\|v_{0}\right\|_{L^{\infty}(\Omega)}+\chi(0)\right) \int_{0}^{t} \int_{\Omega} v_{t} \leqslant \\
& \leqslant 2 \int_{0}^{t} \int_{\Omega}^{t} z^{-1}|\nabla w|^{2}+\mu\left(C_{18}+1\right)^{2} \int_{0} \int_{\Omega} z^{-1}(w-1)^{2}+\mu C_{12} C_{18}^{2} \int_{0}^{t} \int_{\Omega}\left(1-z^{-1}\right)^{2}+ \\
& +\mu C_{12} C_{18}^{2} \int_{0}^{t} \int_{\Omega} v^{2}+2 C_{12} C_{18}^{2}|\Omega|\left(L_{\chi}\left\|v_{0}\right\|_{L^{\infty}(\Omega)}+\chi(0)\right)\left\|v_{0}\right\|_{L^{\infty}(\Omega)} .
\end{aligned}
$$

Using (6.39), (6.46), 6.51) and (6.52) we conclude

$$
\int_{0}^{t}\left|\frac{d}{d t} \int_{\Omega}\left[z^{-1}(w-1)^{2}\right]\right| \leqslant C
$$

The last inequality and 6.51 imply

$$
\lim _{t \rightarrow \infty} \int_{\Omega}\left[z^{-1}(w-1)^{2}\right]=0
$$

From (6.54) and taking into account the following estimate

$$
\|u(\cdot, t)-1\|_{L^{2}(\Omega)}^{2} \leqslant e^{\max _{s \in\left(0,\left\|v_{0}\right\|_{L} \infty(\Omega)\right.} \chi(s)} \int_{\Omega} z^{-1}(w-1)^{2}+\left\|z^{-1}-1\right\|_{L^{2}(\Omega)},
$$

we conclude the proof. 


\section{Acknowledgments}

This work was partially supported by the RTN "Modeling, Mathematical Methods and Computer Simulation of Tumour Growth and Therapy" (MRTN-CT-2004-503661). The first author was also partially supported by project DGES (Spain) Grant MTM2007-61755. The authors would like to thank the Centre de Recerca Matemàtica, where this paper was finished, for the invitation to participate to the research programme on "Mathematical Biology: Modelling and Differential Equations" during February 2009 and for the excellent working conditions provided.

\section{References}

[1] Alikakos N. D., $L^{p}$ bounds of solutions of reaction-diffusion equations, Comm. Partial Differential Equations, 4 (1979), 827-868.

[2] Brezis H., Oswald L., Remarks on sublinear elliptic equations, Nonlinear Anal., 10 (1986), 55-64.

[3] Chaplain M. A. J., Anderson A. R. A., Mathematical modelling of tissue invasion, Cancer Modelling and Simulation, ed. L. Preziosi (Chapmann \& Hall/CRT, 2003), 269-297.

[4] Gilbarg D., Trudinger N.S., Elliptic partial differential equations of second order, Springer, Heidelberg 1983.

[5] Henry D., Geometric theory of semilinear parabolic equations, Lecture Notes Math. 840, Springer, 1981.

[6] Horstmann D., Winkler M., Boundedness vs. blow-up in a chemotaxis system, J. Differ. Eq., 215 (2005), 52-107.

[7] Ladyženskaja O. A., Solonnikov V. A., Ural'ceva N. N., Linear and Quasi-linear Equations of Parabolic Type, Translation of Mathematical Monographs, vol. 23, American Mathematical Society, 1968.

[8] Liţcanu G., Morales-Rodrigo C., Global solutions and asymptotic behavior for a parabolic degenerate coupled system arising from biology, to appear in Nonlinear Analysis: Theory, Methods \& Applications (arXiv:0902.4531).

[9] Osaki K., Tsujikawa T., Yagi A., Mimura M., Exponential attractor for a chemotaxis-growth system of equations, Nonlinear Analysis, 51 (2002), 119-144.

[10] Perumpanani A. J., Byrne H. M., Extracellular matrix concentration exerts selection pressure on invasive cells, European Journal of Cancer, 35 (1999), 1274-1280.

[11] Tao Y., Zhu G., Global solution to a model of tumor invasion, Appl. Math. Sci., 1, no. 48 (2007), $2385-2398$.

[12] Walker C., Webb G.F., Global existence of classical solutions for a haptotaxis model, SIAM J. Math. Anal., 38 (2007), 1694-1713.

[13] Yagi A., Norm behavior of solutions to a parabolic system of chemotaxis, Math. Japonica, 45 (1997), 241-265. 